\title{
Genetic correlations among psychiatric and immune-related phenotypes based on genome-wide association data
}

\author{
Daniel S. Tylee ${ }^{1}$ @ | Jiayin Sun ${ }^{1}$ | Jonathan L. Hess ${ }^{1}$ | Muhammad A. Tahir ${ }^{1}$ | Esha Sharma ${ }^{1}$ |
}

Rainer Malik $^{2}$ | Bradford B. Worrall ${ }^{3}$ (1) | Andrew J. Levine ${ }^{4}$ | Jeremy J. Martinson ${ }^{5}$ |

Sergey Nejentsev ${ }^{6}$ | Doug Speed ${ }^{7}$ | Annegret Fischer ${ }^{8}$ | Eric Mick ${ }^{9}$ | Brian R. Walker ${ }^{10}$ |

Andrew Crawford ${ }^{10,11}$ | Struan F. A. Grant ${ }^{12,13,14,15}$ | Constantin Polychronakos ${ }^{16}$ |

Jonathan P. Bradfield ${ }^{12,17}$ | Patrick M. A. Sleiman ${ }^{12,14}$ | Hakon Hakonarson ${ }^{12,14}$ |

Eva Ellinghaus $^{8}$ | James T. Elder ${ }^{18}$ | Lam C. Tsoi ${ }^{18,19}$ | Richard C. Trembath ${ }^{20}$ |

Jonathan N. Barker ${ }^{20}$ | Andre Franke ${ }^{8}$ | Abbas Dehghan ${ }^{21}$ | The 23 and Me Research

Team $^{22,23}$ | The Inflammation Working Group of the CHARGE Consortium | The

METASTROKE Consortium of the International Stroke Genetics Consortium | The

Netherlands Twin Registry | The neuroCHARGE Working Group | The Obsessive

Compulsive and Tourette Syndrome Working Group of the Psychiatric Genomics

Consortium | Stephen V. Faraone ${ }^{1,23}$ | Stephen J. Glatt ${ }^{1}$

\footnotetext{
${ }^{1}$ Psychiatric Genetic Epidemiology \& Neurobiology Laboratory (PsychGENe Lab), Departments of Psychiatry and Behavioral Sciences \& Neuroscience and Physiology, SUNY Upstate Medical University, Syracuse, New York

${ }^{2}$ Institute for Stroke and Dementia Research, Klinikum der Universität München, Ludwig-Maximilians-University (LMU), Munich, Germany

${ }^{3}$ Departments of Neurology and Public Health Sciences, University of Virginia School of Medicine, Charlottesville, Virginia

${ }^{4}$ Department of Neurology, David Geffen School of Medicine, University of California Los Angeles, Los Angeles, California

${ }^{5}$ Department of Infectious Diseases and Microbiology, Graduate School of Public Health, University of Pittsburgh, Pennsylvania

${ }^{6}$ Department of Medicine, University of Cambridge, Cambridge, United Kingdom

${ }^{7}$ Genetics Institute, University College London, WC1E 6BT, London, United Kingdom

${ }^{8}$ Institute of Clinical Molecular Biology, Christian Albrechts University of Kiel, Kiel, Germany

${ }^{9}$ Department of Quantitative Health Sciences, University of Massachusetts Medical School, Worcester, Massachusetts

${ }^{10}$ BHF Centre for Cardiovascular Science, Queen's Medical Research Institute, University of Edinburgh, Edinburgh, EH16 4TJ, United Kingdom

${ }^{11}$ School of Social and Community Medicine, MRC Integrated Epidemiology Unit, University of Bristol, Bristol, BS8 2BN, United Kingdom

${ }^{12}$ Center for Applied Genomics, Division of Human Genetics, The Children's Hospital of Philadelphia, Philadelphia, Pennsylvania

${ }^{13}$ Division of Endocrinology and Diabetes, The Children's Hospital of Philadelphia, Philadelphia, Pennsylvania

${ }^{14}$ Department of Pediatrics, Perelman School of Medicine, University of Pennsylvania, Philadelphia, Pennsylvania

${ }^{15}$ Institute of Diabetes, Obesity and Metabolism, Perelman School of Medicine, University of Pennsylvania, Philadelphia, Pennsylvania

${ }^{16}$ Endocrine Genetics Laboratory, Department of Pediatrics and the Child Health Program of the Research Institute, McGill University Health Centre, Montreal, Quebec, Canada

${ }^{17}$ Quantinuum Research LLC, San Diego, California

${ }^{18}$ Department of Dermatology, Veterans Affairs Hospital, University of Michigan, Ann Arbor, Michigan
}

Abbreviations: ADHD, attention deficit-hyperactivity disorder; BD, bipolar disorder; BH, Benjamini-Hochberg; CD, Crohn's disease; CRP, C-reactive protein; GWAS, genome-wide association study; GW hits, genome-wide significant associations; HESS, heritability estimation from summary statistics; LD, linkage disequilibrium; LDSC, linkage disequilibrium score regression; MHC, major histocompatibility; OCD, obsessive compulsive disorder; PBC, primary biliary cirrhosis; PGC, Psychiatric Genomics Consortium; QC, quality control; REML, restricted maximum likelihood; SLE, systemic lupus erythematosus; SNP, single nucleotide polymorphism; SZ, schizophrenia; UC, ulcerative colitis. 
${ }^{19}$ Department of Biostatistics, University of Michigan, Ann Arbor, Michigan

${ }^{20}$ Division of Genetics and Molecular Medicine, King's College London, London, UK

${ }^{21}$ Department of Biostatistics and Epidemiology, MRC-PHE Centre for Environment and Health School of Public Health, Imperial College London, London, United Kingdom

${ }^{22} 23 a n d M e$, Inc., Mountain View, California

${ }^{23}$ K.G. Jebsen Centre for Research on Neuropsychiatric Disorders, University of Bergen, Bergen, Norway

Correspondence: Daniel S. Tylee, SUNY Upstate Medical University, 750 East Adams Street, Syracuse, NY 13210.

Email: dantylee@gmail.com

Funding information

Autism Speaks, Grant/Award Number: Weatherstone Pre-doctoral Training Grant \#9645; DS; Brain and Behavior Research Foundation, Grant/Award Number: SJG; Gerber Foundation, Grant/Award Number: SJG; National Alliance for Research on Schizophrenia and Depression, Grant/Award Number: SJG; National Institutes of Health, Grant/Award Number: R01MH101519, R01AG054002, P50MH081755 (SJG), R03DA; NIHR Cambridge Biomedical Research Centre, Grant/Award Number: SN; Sidney R. Baer, Jr. Foundation, Grant/Award Number: SJG; UCLA Center for AIDS Research, Grant/Award Number: Al2869; AJL; Vascular Dementia Research Foundation, Grant/Award Number: RM; Wellcome Trust, Grant/Award Number: SN
Individuals with psychiatric disorders have elevated rates of autoimmune comorbidity and altered immune signaling. It is unclear whether these altered immunological states have a shared genetic basis with those psychiatric disorders. The present study sought to use existing summary-level data from previous genome-wide association studies to determine if commonly varying single nucleotide polymorphisms are shared between psychiatric and immune-related phenotypes. We estimated heritability and examined pair-wise genetic correlations using the linkage disequilibrium score regression (LDSC) and heritability estimation from summary statistics methods. Using LDSC, we observed significant genetic correlations between immune-related disorders and several psychiatric disorders, including anorexia nervosa, attention deficit-hyperactivity disorder, bipolar disorder, major depression, obsessive compulsive disorder, schizophrenia, smoking behavior, and Tourette syndrome. Loci significantly mediating genetic correlations were identified for schizophrenia when analytically paired with Crohn's disease, primary biliary cirrhosis, systemic lupus erythematosus, and ulcerative colitis. We report significantly correlated loci and highlight those containing genome-wide associations and candidate genes for respective disorders. We also used the LDSC method to characterize genetic correlations among the immune-related phenotypes. We discuss our findings in the context of relevant genetic and epidemiological literature, as well as the limitations and caveats of the study.

\section{KEYWORDS}

allergy, anorexia nervosa, attention deficit-hyperactivity disorder, autoimmune disorder, bipolar disorder, celiac disease, childhood ear infection, C-reactive protein, Crohn's disease, genetic correlation, genome-wide association, hypothyroidism, major depression, neuroticism, obsessive schizophrenia, primary biliary cirrhosis, rheumatoid arthritis, smoking, systemic lupus erythematosus, Tourette syndrome, tuberculosis susceptibility, type 1 diabetes, ulcerative colitis

\section{1 | INTRODUCTION}

The biological bases of major psychiatric disorders have been studied for decades, yet they remain largely unresolved. Evidence from both clinical and biomedical literature has demonstrated that individuals with these conditions show differences in circulating immunologic markers, functional capacities of isolated immune cells, and atypical prevalence of clinical immune-related phenotypes compared to individuals not affected by psychiatric or neurodevelopmental disorders (Dowlati, 2010; Eaton et al., 2006; Fineberg \& Ellman, 2013; Gesundheit et al., 2013; Gibney \& Drexhage, 2013; Hess et al., 2016; Jones \& Thomsen, 2013; Masi et al., 2015; Modabbernia, Taslimi, Brietzke, \& Ashrafi, 2013; Rege \& Hodgkinson, 2013). It remains unclear what roles (if any) altered immunologic functions may play in the major psychiatric phenotypes, though plausible mechanisms linking altered immune functions with neurobiological changes during early brain development and in fully developed adults have been identified (Deverman \& Patterson, 2009; Felger \& Lotrich, 2013; Meyer, 2014;
Miller, Haroon, Raison, \& Felger, 2013; Oskvig et al., 2012; Sekar et al., 2016; Shatz, 2009; Smith, Li, Garbett, Mirnics, \& Patterson, 2007). While some studies have already suggested potential genetic bases for the immune dysregulation observed in a subset of psychiatric patients (Jung, Kohane, \& Wall, 2011; Stringer, Kahn, de Witte, Ophoff, \& Derks, 2014; The Network \& Pathway Analysis Subgroup of the Psychiatric Genomics Consortium, 2015; Wang, Yang, Gelernter, \& Zhao, 2015), the extent to which co-occurrence or segregation of clinical phenotypes may be influenced by similarities in genomewide genetic risk signals warrants further examination. Genome-wide association studies (GWASs) and meta-analyses can shed light on the regions of the genome that tend to associate with a clinical phenotype, quantitative trait, or biomarker; this is accomplished through tagging and association-testing of single nucleotide polymorphisms (SNPs) that vary within the population. Recently developed methods like linkage disequilibrium (LD) score regression (LDSC; Bulik-Sullivan, Finucane, et al., 2015a) and heritability estimation from summary statistics (HESS; Shi, Mancuso, Spendlove, \& Pasaniuc, 2017) allow for 
direct comparison of GWAS summary statistics for two different phenotypes for quantitative assessment of genetic correlation.

In the present study, we leveraged existing data to explore the genetic associations of a set of medical phenotypes that are enriched with immune and inflammatory processes; these included allergic conditions, classic autoimmune diseases, other inflammatory diseases, and vulnerability to infectious disease. We sought to crosscorrelate the genetic associations of these phenotypes with the associations obtained from studies of a set of psychiatric and behavioral phenotypes. We hypothesized that some phenotype-pairs with evidence for increased clinical comorbidity might also share similarities in their genome-wide association profile, which would be reflected in our analyses as significant positive correlations. Additionally, in light of literature suggesting shared genetic risk among some immune and inflammatory disorders, we assessed genetic correlations within this set of phenotypes using the LDSC method; these findings are reported within the Supporting Information. Genetic correlations within the set of psychiatric phenotypes have been reported previously (Anttila, 2016; Bulik-Sullivan, Finucane, et al., 2015a; Zheng et al., 2016) and are not examined in the present study.

\section{2 | MATERIALS AND METHODS}

\section{1 | Literature search}

We searched the published literature (Pubmed, SCOPUS), data repositories (dbGaP and immunobase.org), and the downloads page of the Psychiatric Genomics Consortium (PGC) website (https://www.med. unc.edu/pgc/downloads) to identify phenotypes with potentially usable GWAS and GWAS meta-analysis summary statistics. For studies identified in the published literature, we contacted corresponding authors to request summary statistics. In order to facilitate crossstudy comparison, we utilized studies that reported samples of European ancestry, broadly defined to include Central, Southern and Eastern Europe, Scandinavia, and Western Russia. Our initial search yielded a large number of datasets reflecting a wide range of behavioral and immune-related phenotypes (Supporting Information Table S1); the set of phenotypes ultimately retained for final analyses was selected based on criteria described below. When multiple studies were identified for a given phenotype, we pursued the studies with the largest effective sample sizes and ultimately used the available study with the largest heritability z-score. In several instances, data from the largest existing studies could not be shared or reflected a mixed-ancestry meta-analysis; in these cases, we deferred to the next largest European-ancestry study. We chose to retain datasets with an effective sample size greater than 5,000 individuals and with estimated SNP heritability $z$-score $\geq 3$, in keeping with previous recommendations (Bulik-Sullivan, Finucane, et al., 2015a). This filter resulted in the exclusion of many relevant immune-related phenotypes, including eosinophilic esophagitis (Sleiman et al., 2014), granulomatosis with polyangiitis (Xie et al., 2013), IgA nephropathy (Kiryluk et al., 2014), HIV-related neurocognitive phenotypes (Levine et al., 2012), morning cortisol levels (Bolton, 2014), myeloid leukemias (Tapper et al., 2015), psoriatic arthritis (Ellinghaus et al., 2012), sarcoidosis (Fischer et al., 2012), and systemic sclerosis (Radstake et al., 2010). This also resulted in exclusion of several psychiatric and behavior phenotypes, including adolescent alcohol abuse (Edwards et al., 2015), anxiety-spectrum disorders (Otowa et al., 2016), borderline personality disorder (Lubke et al., 2014), language impairment (Jernigan et al., 2016), personality domains (five factor model; de Moor et al., 2012), post-traumatic stress disorder (Duncan, 2017a), and reading disability (Eicher et al., 2013). We also ultimately excluded data from studies of ethanol, opiate, and cocaine dependence (Gelernter, Kranzler, Sherva, Almasy, et al., 2014a; Gelernter, Kranzler, Sherva, Koesterer, et al., 2014b; Gelernter, Sherva, et al., 2014c), as genetic correlations involving these phenotypes were frequently outside the boundaries tolerated by the LDSC software, making them difficult to interpret; this may have been related to the ordinal-ranked phenotypes used in the GWASs. Finally, while relationships between tobacco-smoking behavior and other psychiatric phenotypes have been examined previously (Bulik-Sullivan, Finucane, et al., 2015a; Zheng et al., 2016), we chose to retain smoking data in order to assess relationships with a more complete set of immunerelated phenotypes. The full list of phenotypes identified in the search and retained for analyses is shown in Supporting Information Table S1, along with identification of the study cohorts and consortia that generated these data, full citations of the respective publications, and indications of sample size, information regarding genomic inflation, and estimated SNP heritability.

\section{2 | GWAS phenotypes retained for genetic correlation}

For our psychiatric and behavior-related phenotypes, we ultimately retained GWAS summary data reflecting studies of Alzheimer's disease (Lambert et al., 2013), angry temperament (Mick et al., 2014), anorexia nervosa (Duncan et al., 2017b), attention deficithyperactivity disorder (ADHD; Demontis, 2017), autism (Anney, 2017), bipolar disorder (BD; Hou et al., 2016; Sklar et al., 2011), cigarette smoking (ever-smoked status; The Tobacco \& Genetics Consortium, 2010), major depressive disorder (Ripke et al., 2013), trait neuroticism (Turley et al., 2018), obsessive-compulsive disorder (OCD; Arnold, 2017), Parkinson's disease (Pickrell et al., 2016), schizophrenia (SZ; Ripke, 2014), and Tourette Syndrome (personal communication from PGC Working Group). Collectively, these phenotypes were treated as a set. For phenotypes that are known or suspected to involve alterations to immune cells and/or inflammatory signaling, we ultimately retained GWAS data reflecting allergy (any, self-reported; Hinds et al., 2013; Pickrell et al., 2016), asthma (selfreported; Pickrell et al., 2016), atopic dermatitis (EArly Genetics and Lifecourse Epidemiology (EAGLE) Eczema Consortium, 2015), childhood ear infection (self-reported; Pickrell et al., 2016), celiac disease (Dubois, 2010), serum C-reactive protein (CRP; Dehghan, 2011), Crohn's disease (CD; Franke et al., 2010; Liu et al., 2015), hypothyroidism (self-reported; Pickrell et al., 2016), primary biliary cirrhosis (PBC; Cordell, 2015), psoriasis (Tsoi et al., 2015), rheumatoid arthritis (Okada et al., 2014), systemic lupus erythematosus (SLE; Bentham et al., 2015), susceptibility to pulmonary tuberculosis (Curtis et al., 2015), type 1 diabetes (Bradfield et al., 2011), and ulcerative colitis 
(UC; Anderson, 2011; Liu et al., 2015) These phenotypes were treated as a set in subsequent analyses.

\section{3 | Data pre-processing and analysis}

Our primary analyses were performed using the LDSC software (https://github.com/bulik/ldsc; Bulik-Sullivan, Finucane, et al., 2015a). Briefly, this set of tools can be used with existing GWAS summary data in order to distinguish polygenicity from confounding caused by uncontrolled population stratification or cryptic relatedness among samples (Bulik-Sullivan, Loh, et al., 2015b), to estimate the heritability of a given phenotype (Bulik-Sullivan, Finucane, et al., 2015a), and to estimate the genetic correlation between two phenotypes based on two separate or related sets of summary statistics (Bulik-Sullivan, Finucane, et al., 2015a). In the latter application, the minimal requirements for each set of summary statistics include columns of data indicating SNP ID, the identities of reference and nonreference alleles, association $p$-value, effect size, test statistic (e.g., odds ratio, regression $\beta$, or Z-score), and sample size (per SNP or for all SNPs). For each pair of phenotypes, this tool compares the strength and direction of association signal at each locus while correcting for the correlation that would be expected based on genetic linkage alone, and it provides an estimate of the genetic correlation between phenotypes. This method relies on adjustment for the linkage between SNPs (i.e., covariance caused by genomic proximity); for our analyses, we used the set of LD scores provided by the software's creators, based on the 1000 Genomes Project's European sample (file = eur_w_ld_chr, URL = https://data.broadinstitute.org/alkesgroup/ LDSCORE). Because minor allele frequencies (MAFs) and imputation quality scores were not available for all the obtained sets of GWAS results, we filtered the GWAS results to retain only SNPs that were included within the HapMap3 panel and had a MAF $\geq 5 \%$ within the 1000 Genomes Project Phase 3 European samples (Bulik-Sullivan, Finucane, et al., 2015a); this decision resulted in the exclusion of a sizable proportion of SNPs, but ensured equitable treatment of all datasets. The extended major histocompatibility complex (MHC) region contains high amounts of long-range LD, making it challenging to accurately map association signals in this region. For this reason, and following the work of others (Bulik-Sullivan, Finucane, et al., 2015a; Zheng et al., 2016), we excluded this region from our analyses (chromosome 6, base positions $25 \times 10^{6}$ to $\left.35 \times 10^{6}\right)$. Additional SNP quality control (QC) routines followed those implemented by the GWAS authors and the defaults employed with the LDSC munge_sumstats.py function; this function checks alleles to ensure that the supplied alleles match those in the HapMap3 reference panel. For each dataset, we estimated the phenotype's heritability. The results of this analysis, along with features of each GWAS dataset (sample size, number of QC-positive SNPs, genomic inflation factor, etc.), are shown for all phenotypes in Supporting Information Table S1. All phenotypes with sample size $\geq 5,000$ and estimated SNP heritability $z$-score $\geq 3$ were retained for correlation analysis (indicated in Supporting Information Table S1 in green highlight). Pair-wise genetic correlations were assessed between retained phenotypes based on the intersection of QC-positive SNPs, and heatmaps were constructed to depict these relationships. For correlation coefficients returned within the bounds of the LDSC software, $p$-values were corrected using the BenjaminiHochberg $(\mathrm{BH})$ method for the total number of unique tests depicted in each correlation matrix. Within the main text, we describe only correlations that survived family-wise multiple-test correction. Correlations are reported as the coefficient \pm standard error. For phenotypepairs showing statistically significant genetic correlations, we reevaluated the genetic correlations and estimated heritability using the HESS method (https://github.com/huwenboshi/hess; Shi et al., 2017)

\subsection{Characterization of genetically correlated loci and associated genes}

For psychiatric-immune phenotype-pairs showing significant genetic correlations after $\mathrm{BH}$ correction for multiple testing, we used the HESS software to estimate partitioned heritability and genetic correlations based on smaller LD-based segments of the genome (average size $=1.5 \mathrm{Mb}$ ). We report the number and identity of genomic partitions (based on HG19 reference) displaying significant local genetic correlations and apply correction for the total number of partitions ( $\approx 1,694$, after MHC removal). Because presently available methods are poorly suited for fine-mapping the loci mediating a genetic correlation, we prioritized reporting correlated loci that also contain genome-wide significant associations for the relevant phenotypes (i.e., associations with $p<5 \times 10^{-8}$; subsequently called GW hits). We report GW hits contained within the present datasets, but also cross-reference these findings with those contained in immunobase.org, in order to identify loci associated with multiple immune-related disorders. We report the HGNC symbols for candidate genes proposed to mediate those associations. The full list of genes contained within each correlated loci is provided in Supporting Information Table S3. Additionally, we used HESS to examine patterns of local genetic correlation in relationship to GWAS hits to make inferences about putative causal directionality between the phenotype-pairs. For all HESS analyses, we used the 1000 Genomes Project Phase 3 European reference panel and the LD-independent genome partitions recommended by the software developers (Berisa \& Pickrell, 2015). Following the developers' practices, we assumed no sample overlap for comparisons of data generated by different consortia (Shi et al., 2017).

\section{3 | RESULTS}

\section{1 | Genome-wide correlations between psychiatric and immune-inflammatory phenotypes}

All pair-wise LDSC genetic correlations between psychiatric and immune-related phenotypes are depicted in Figure 1. Notably, 21 correlations survived $\mathrm{BH}$ correction for multiple testing (denoted with **) and 6 survived a more stringent Bonferroni correction (denoted with ***). Full results for these analyses are provided in Supporting Information Table S2. Significant positive relationships were observed between ADHD and each of: CRP ( $r g=0.23 \pm 0.06, p=2.0$ $\left.\times 10^{-4}\right)$, childhood ear infections $\left(r g=0.20 \pm 0.05, p=2.0 \times 10^{-4}\right)$, psoriasis $\left(r g=0.23 \pm 0.07, p=1.0 \times 10^{-3}\right)$, rheumatoid arthritis $(r g=$ 


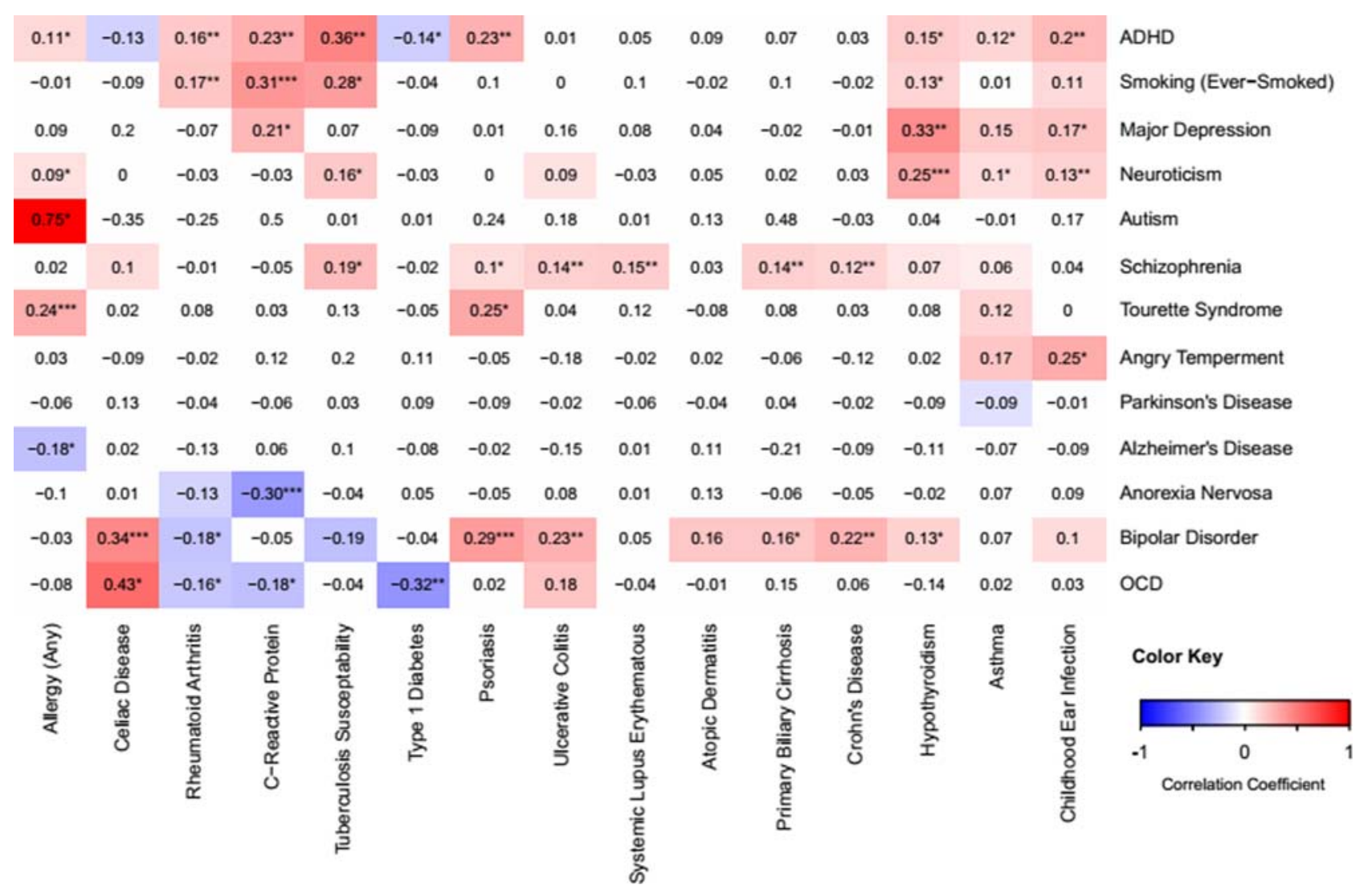

FIGURE 1 A heatmap depicting LDSC genome-wide genetic correlations between psychiatric and immune-related conditions such that red reflects more positive correlation coefficients while blue reflects more negative coefficients. Correlation coefficients are provided within each cell, with full details provided in Supporting Information Table S2. Correlations reaching trend-level significance $(.05<$ uncorrected $p<.10)$ are depicted as colored panels, while relationships surpassing uncorrected $p<.05$ are additionally denoted with *, and relationships surpassing $\mathrm{BH}-p<.05$ (for the total number of tests depicted in the figure) are denoted with **. The rows and columns of the heatmap are hierarchically clustered based on correlation coefficients. ADHD = attention deficit-hyperactivity disorder; OCD = obsessive-compulsive disorder [Color figure can be viewed at wileyonlinelibrary.com]

$\left.0.16 \pm 0.05, p=9.0 \times 10^{-4}\right)$, and tuberculosis susceptibility $(r g=$ $\left.0.36 \pm 0.11, p=1.6 \times 10^{-3}\right)$. Anorexia nervosa showed a negative genetic correlation with CRP $\left(r g=-0.30 \pm 0.08, p=1.0 \times 10^{-4}\right)$. BD was positively correlated with each of: celiac disease $(r g=0.31 \pm$ $\left.0.09, p=4.0 \times 10^{-4}\right), \mathrm{CD}\left(r g=0.21 \pm 0.05, p=3.7 \times 10^{-5}\right)$, psoriasis $\left(r g=0.25 \pm 0.08, p=3.8 \times 10^{-3}\right)$, and UC $(r g=0.23 \pm 0.06, p=2.0$ $\times 10^{-4}$ ). Major depressive disorder was positively correlated with hypothyroidism $\left(0.33 \pm 0.09, p=5.0 \times 10^{-4}\right)$. Similarly, neuroticism was positively correlated with hypothyroidism ( $r g=0.25 \pm 0.06, p=$ $\left.7.2 \times 10^{-5}\right)$, in addition to childhood ear infection $(r g=0.13 \pm 0.04$, $\left.p=8.0 \times 10^{-4}\right) . O C D$ was negatively correlated with type 1 diabetes $\left(r g=-0.32 \pm 0.11, p=5.4 \times 10^{-3}\right)$. Smoking behavior was positively correlated with CRP $\left(r g=0.31 \pm 0.07, p=3.6 \times 10^{-5}\right)$ and with rheumatoid arthritis $\left(r g=0.17 \pm 0.05, p=2.3 \times 10^{-3}\right)$. SZ showed positive genetic correlations with $\mathrm{CD}(r g=0.12 \pm 0.03, p=2.0 \times$ $\left.10^{-4}\right)$, PBC $\left(r g=0.14 \pm 0.05, p=2.0 \times 10^{-3}\right)$, SLE $(r g=0.15 \pm 0.04$, $\left.p=2.0 \times 10^{-4}\right)$, and UC $\left(r g=0.14 \pm 0.04, p=2.0 \times 10^{-4}\right)$. Finally, we observed a positive genetic correlation between Tourette syndrome and allergy $\left(r g=0.24 \pm 0.06\right.$, uncorrected $\left.p=2.7 \times 10^{-5}\right)$. Additionally, several large-magnitude correlations attained a nominal threshold of statistical significance (e.g., autism-allergy and OCD-celiac); these correlations tended to have a higher standard error and were generated using relatively smaller GWAS sample sizes. As such, they may be more likely to reflect false positives and should be regarded with appropriate skepticism.

For phenotypes involved in correlations that survived multiple test correction, estimated SNP heritability is shown in Table 2. For these phenotypes, we reassessed SNP heritability and the magnitude of genome-wide genetic correlations using the HESS method (Tables 1 and 2). Correlation coefficients were not correlated between the two methods (Pearson $r=0.25, p=0.25$; Supporting Information Figure $\mathrm{S} 1$ ) and the absolute value of the difference was negatively related to sample size $(r=-0.45, p=0.035$; Supporting Information Figure S2), which is consistent with the software developer's guidelines (Shi et al., 2017). LDSC-based correlations among the immunerelated phenotypes are reported in the Supporting Information Text and Table S5.

\section{2 | Characterization of loci contributing to psychiatric-immune genetic correlations}

For psychiatric-immune phenotype-pairs that demonstrated a significant genome-wide correlation with the LDSC method (i.e., those in Table 1), we used the HESS software to examine the genetic correlation within the $\sim 1,694$ partitioned genomic loci. The number of correlated loci before and after $\mathrm{BH}$ multiple test correction are depicted 
TABLE 1 Significant genome-wide psychiatric-immune genetic correlations

\begin{tabular}{|c|c|c|c|}
\hline $\begin{array}{l}\text { Psychiatric } \\
\text { phenotype }\end{array}$ & $\begin{array}{l}\text { Immune-related } \\
\text { phenotype }\end{array}$ & $\begin{array}{l}\text { LDSC correlation } \pm \text { error, } \\
\text { uncorrected } p \text {-value }\end{array}$ & HESS correlation \pm error \\
\hline ADHD & Childhood ear infection & $0.20 \pm 0.05, p=2.0 \times 10^{-4}$ & $0.14 \pm 0.03$ \\
\hline ADHD & Rheumatoid arthritis & $0.16 \pm 0.05, p=9.0 \times 10^{-4}$ & $0.29 \pm 0.04$ \\
\hline ADHD & Tuberculosis susceptibility & $0.36 \pm 0.11, p=1.6 \times 10^{-3}$ & $0.87 \pm 0.25$ \\
\hline BD & $C D$ & $0.22 \pm 0.06, p=5.0 \times 10^{-4}$ & $1.31 \pm 0.07$ \\
\hline BD & Psoriasis & $0.29 \pm 0.07, p=2.7 \times 10^{-5}$ & $5.76 \pm 0.58$ \\
\hline BD & UC & $0.23+0.07, p=1.5 \times 10^{-3}$ & $1.59 \pm 0.08$ \\
\hline Cigarettes (ever-smoked) & CRP & $0.31 \pm 0.07, p=3.6 \times 10^{-5}$ & $2.24 \pm 0.73$ \\
\hline Neuroticism & Hypothyroidism & $0.25 \pm 0.06, p=7.2 \times 10^{-5}$ & $0.03 \pm 0.01$ \\
\hline OCD & Type 1 diabetes & $-0.32+0.11, p=5.4 \times 10^{-3}$ & $0.98 \pm 0.18$ \\
\hline SZ & $C D$ & $0.12 \pm 0.03, p=2.0 \times 10^{-4}$ & $0.31 \pm 0.03$ \\
\hline SZ & PBC & $0.14 \pm 0.05, p=2.0 \times 10^{-3}$ & $0.88 \pm 0.05$ \\
\hline SZ & SLE & $0.15 \pm 0.05, p=1.2 \times 10^{-3}$ & $0.12 \pm 0.02$ \\
\hline SZ & UC & $0.14 \pm 0.04, p=2.0 \times 10^{-4}$ & $0.56 \pm 0.03$ \\
\hline Tourette's syndrome & Allergy (any) & $0.24 \pm 0.06, p=2.7 \times 10^{-5}$ & $0.29 \pm 0.07$ \\
\hline
\end{tabular}

Note. This table displays psychiatric-immune phenotype-pairs showing genome-wide genetic correlation with the linkage disequilibrium score regression (LDSC) method after correction for the total number of genetic correlations depicted in Figure 1 using the Benjamini-Hochberg (BH) method. We also report the genome-wide correlation estimates produced by the heritability estimation from summary statistics $(H E S S)$ method. ADHD $=$ attention deficit-hyperactivity disorder; $\mathrm{BD}=$ bipolar disorder; $\mathrm{CD}=$ Crohn's disease; $\mathrm{CRP}=\mathrm{C}$-reactive protein; $\mathrm{OCD}=$ obsessive compulsive disorder; $\mathrm{PBC}=$ primary biliary cirrhosis; SLE = systemic lupus erythematosus; SZ = schizophrenia; UC = ulcerative colitis.

in Table 3; detailed results for these analyses, including local heritability, correlation strength, and the lists of gene symbols within each loci are provided in Supporting Information Table S3. Only SZ displayed robust local genetic correlations with immune-related phenotypes, including thirty-two loci with CD, 37 loci with PBC, 20 loci with SLE, and 8 with UC (Table 3, depicted in Figure 2). Upon closer examination of the loci implicated between SZ and CD, we noticed that five of these loci contained GW hits, including one locus on chromosome 4q24 (4:100678360-103221356; highlighted green in Figure 2) that contained GW hits for both SZ and CD within the present data, and with four other autoimmune diseases (immunobase. org); these signals are near autoimmunity candidate genes NFKB1 and MANBA, as well as proposed SZ candidate gene SLC39A8, among others contained within the locus (see Supporting Information Table S3). The locus on 10p12.3 (10:18725659-18816236, highlighted green) contains a GW hit for SZ attributed to calcium channel gene CACNB2. Another locus mediating a significantly correlated locus on 12q12 (12:39227169-40816185, highlighted green) contains a GW hit for CD attributed to LRRK2. When examining the loci implicated between SZ and PBC, we observed three harboring GW hits for the former and three harboring signals for the latter, including loci within 3p24.3 (3:16282442-17891118, highlighted orange) containing PLCL2 and within 11q23.3 (11:117747110-119215476, highlighted orange), containing candidate genes CXCR5, DDX6, and TREH. Among the loci implicated between SZ and SLE, we observed two harboring GW hits for the former and three harboring hits for the latter. One such locus within 1q21 (1:148361253-151538881, highlighted yellow) contains a SZ association signal localizing near candidate gene APH1A. Another locus within 1q23 (1:159913048-162346721, highlighted yellow) contains a GW hit for SLE, as well as several other autoimmune diseases, associated with candidate gene FCGR2A. Similarly, a locus within 22q11.21 (22:19912358-22357325, highlighted yellow) containing multidisease association signal is associated with MAPK1 and UBE2L3. Among the loci implicated between SZ and UC, one within 11q13.1 (11:63804569-65898631) harbored GW hits for multiple autoimmune disorders.

We also sought to examine whether the specific loci might be implicated across multiple psychiatric-immune disorder pairs (Figure 2). An analysis limited to only those surviving $\mathrm{BH}$ correction for multiple testing yielded only two loci shared by multiple disease pairs. The first locus (within 3p24.3; 3:21643707-22204244) was identified in correlations of SZ with PBC and with CD; it contained no GWS hits and two genes of unclear consequence ZNF385D and ZNF385D-AS2. The second locus within 8p32.1 (8:1127899813491775 , highlighted brown) was identified in correlations of SZ with PBC and with SLE; this locus contained numerous genes and is adjacent to a GWS hit for SLE associated with candidate gene BLK. When we broadened the scope to examine all loci implicated in nominally significant correlations (uncorrected $p<0.05$ ), we find several that are common to multiple psychiatric-immune disorder pairs 
TABLE 2 Sample characteristics for phenotypes involved in significant correlations

\begin{tabular}{|c|c|c|c|c|}
\hline Phenotype & Data source & $\begin{array}{c}\text { Estimated genome-wide SNP } \\
\text { heritability } \pm \text { error } \\
\text { (LDSC/HESS) }\end{array}$ & GWAS N & $\begin{array}{c}\text { QC-Positive SNPS } \\
\text { (MHC Excluded) }\end{array}$ \\
\hline ADHD & Demontis (2017) & $0.24 \pm 0.02 / 0.26 \pm 0.02$ & 53,293 & $1,004,958$ \\
\hline Allergy (any, self-report) & The 23andMe Research Team & $0.08 \pm 0.01 / 0.15 \pm 0.01$ & 181,000 & $1,060,611$ \\
\hline Anorexia nervosa & Duncan et al. (2017b) & $0.26 \pm 0.04 / 0.09 \pm 0.04$ & 14,477 & $1,054,719$ \\
\hline $\mathrm{BP}$ & Hou et al. (2016) & $0.20 \pm 0.02 / 0.14+0.02$ & 40,225 & $1,052,397$ \\
\hline Childhood ear infection & The 23andMe Research Team & $0.07 \pm 0.01 / 0.10 \pm 0.01$ & 122,000 & $1,060,612$ \\
\hline Celiac disease & Dubois (2010) & $0.30 \pm 0.05 / 0.13 \pm 0.04$ & 15,283 & 271,764 \\
\hline Cigarettes (ever-smoked) & Tobacco and Genetics Consortium & $0.07 \pm 0.01 / 0.01 \pm 0.02$ & 74,035 & 963,355 \\
\hline$C D$ & Liu et al. (2015) & $0.47 \pm 0.06 / 0.33 \pm 0.03$ & 21,389 & $1,062,075$ \\
\hline CRP & Dehghan (2011) & $0.13+0.02 / 0.11 \pm 0.02$ & 66,185 & 965,855 \\
\hline Hypothyroidism (self-report) & The 23andMe Research Team & $0.05+0.01 / 0.08 \pm 0.01$ & 135,000 & $1,060,612$ \\
\hline Major depression & PGC Depression Working Group & $0.14 \pm 0.03 / 0.07 \pm 0.04$ & 18,759 & 967,534 \\
\hline Neuroticism & Social Science Genetics Consortium & $0.09 \pm 0.01 / 0.44 \pm 0.01$ & 168,105 & $1,053,712$ \\
\hline OCD & PGC OCD/TS Working Group & $0.29 \pm 0.05 / 0.09 \pm 0.04$ & $10,215^{*}$ & $1,054,746$ \\
\hline PBC & Cordell (2015) & $0.37+0.06 / 0.17 \pm 0.04$ & 13,239 & 940,715 \\
\hline Psoriasis & Tsoi et al. (2015) & $0.82 \pm 0.13 / 0.09 \pm 0.04$ & $5,116 *$ & $1,037,355$ \\
\hline Rheumatoid arthritis & Okada et al. (2014) & $0.14+0.02 / 0.10 \pm 0.01$ & 58,284 & $1,051,805$ \\
\hline SZ & PGC Schizophrenia Working Group & $0.47 \pm 0.02 / 0.62 \pm 0.01$ & 77,096 & $1,061,529$ \\
\hline SLE & Bentham et al. (2015) & $0.27 \pm 0.05 / 0.27 \pm 0.03$ & 23,210 & $1,056,783$ \\
\hline Tourette syndrome & PGC OCD/TS Working Group & $0.35 \pm 0.04 / 0.08 \pm 0.05$ & $13,341 *$ & $1,041,689$ \\
\hline Tuberculosis susceptibility & Curtis et al. (2015) & $0.18 \pm 0.05 / 0.02 \pm 0.05$ & 11,936 & 819,917 \\
\hline Type 1 diabetes & Bradfield et al. (2011) & $0.18 \pm 0.03 / 0.15 \pm 0.03$ & 26,890 & 854,164 \\
\hline UC & Liu et al. (2015) & $0.25 \pm 0.03 / 0.23 \pm 0.03$ & 27,432 & $1,062,094$ \\
\hline
\end{tabular}

Note. This table displays phenotype names, data sources, and estimated SNP heritability using the linkage disequilibrium score regression (LDSC) and heritability estimation from summary statistics (HESS) methods, as well as the GWAS sample size and number of SNPs surviving quality control. Full publication references, consortia names, links to web resources, and additional details on the original studies are provided in Supporting Information Table S1. GWAS N denoted with * indicates the median N for all SNPs. ADHD = attention deficit-hyperactivity disorder; BD = bipolar disorder; CD = Crohn's disease; CRP = C-reactive protein; OCD = obsessive compulsive disorder; PBC = primary biliary cirrhosis; PGC = Psychiatric Genomics Consortium; QC = quality control; SNP = single nucleotide polymorphism; SLE = systemic lupus erythematosus; $\mathrm{SZ}=$ schizophrenia; UC = ulcerative colitis.

(Table 4). The most widely implicated locus was shared among the five pairs of psychotic and inflammatory bowel disorders (within 17q12; 17:36809344-38877404, highlighted purple) and contains a GW hit for BD ascribed to candidate gene ERBB2. There were another eight loci that were implicated in four disorder pairs. Among these, one located within 1q32.1 (1:200137649-201589975, highlighted purple) contains GW hits for multiple autoimmune disorders (including celiac disorder, CD, multiple sclerosis, and UC) and is near candidate genes CACNA1S and KIF21B. The full list of loci implicated across multiple disorder pairs is available in Supporting Information Table S3. The results of the HESS analysis of putative causal directionality (Table 5 ) indicated that local genetic correlations were stronger in the loci containing GW hits for SZ $(r g \approx 0.41 \pm 0.12)$ as compared with those containing hits for the paired autoimmune diseases $(r g \approx 0.17 \pm 0.13)$.

\section{4 | DISCUSSION}

In contrast to previous studies examining large sets of medical, anthropomorphic, metabolic, and behavioral phenotypes (Anttila, 2016; Bulik-Sullivan, Finucane, et al., 2015a; Ohn, 2017; Shi et al., 2017; Zheng et al., 2016), the present study performed a focused comparison of psychiatric and immune-related phenotypes using two methods to estimate genetic correlation from summary statistics. We used updated versions of psychiatric GWASs (Anney, 2017; Arnold, 2017; Demontis, 2017; Psychiatric GWAS Consortium Bipolar Disorder Working Group, 2011), and compiled a more comprehensive set of immune-related phenotypes, while simultaneously reducing the burden imposed by multiple testing. Additionally, this analysis reflects the first application of the LDSC and HESS method for some of these phenotype-pairs. We identified several genome-wide correlations that were robust to multiple testing. Furthermore, we used the HESS method to validate genome-wide correlations and to conduct a quantitative analysis that localizes correlations to regions of the genome. We prioritized the reporting of findings based on co-localization with $\mathrm{GW}$ hits. As such, this study provides a quantitative map of genetic relationships between psychiatric and immune-related disorders and serves, along with previous work (Wang et al., 2015), as a starting point for identifying and characterizing potentially pleiotropic loci.

Prominent among the LDSC genome-wide significant findings was a cluster of modest positive correlations involving BD ( $r$ gs ranging 0.25 to 0.33 ) and SZ (rgs ranging 0.12 to 0.15 ) in conjunction with immunerelated disorders involving the gastrointestinal tract (i.e., $C D, P B C, U C$ ). These findings are consistent with available epidemiological evidence indicating that the presence of one set of disorders portends increased risk for a diagnosis from the other class of disorders, though the 
TABLE 3 Significant local genetic correlations based on HESS analysis

\begin{tabular}{|c|c|}
\hline Phenotype pair & $\begin{array}{l}\text { No. of correlated loci }(\mathrm{BH} p<.05 / p<.05) \text { with GWS hits and } \\
\text { associated genes contained within correlated loci }(\mathrm{BH} p<0.05)\end{array}$ \\
\hline ADHD-CEA & $0 / 3$ \\
\hline ADHD-RA & $0 / 5$ \\
\hline ADHD-tuberculosis susceptibility & $0 / 0$ \\
\hline BD-celiac disease & $0 / 30$ \\
\hline $\mathrm{BD}-\mathrm{CD}$ & $0 / 12$ \\
\hline BD -psoriasis & $0 / 3$ \\
\hline BD -UC & $0 / 5$ \\
\hline Cigarettes (ever-smoked)-CRP & $0 / 0$ \\
\hline Neuroticism-HPT & $0 / 15$ \\
\hline OCD-type 1 diabetes & $0 / 1$ \\
\hline SZ-CD & $\begin{array}{l}\text { 32/251 } \\
\text { SZ 4:102921704-103198082** (ACTR3BD4, BDH2, CENPE, SLC39A8, SLC9B1, SLC9B2) } \\
\text { CD 4:103188709-103198082** (CENPE) } \\
\text { CD 8:126529074-126568355 (FAM84B) } \\
\text { CD 10:64301873-64588424 (No Genes) } \\
\text { CD 12:40337163-40815560 (CNTN1, LRRK2, MUC19, RNU6-713P); } \\
\text { CD 21:16790941-16841303 (No Genes) }\end{array}$ \\
\hline SZ-PBC & $\begin{array}{l}37 / 256 \\
\text { SZ 1:30427639-30437268 (No Genes) } \\
\text { SZ 10:18725659-18816236 (AIFM1P1, CACNB2) } \\
\text { PBC 3:16955259-16955259** (PLCL2) } \\
\text { PBC 11:118579747-118743772** (ARCN1, CXCR5, DDX6, MIR6716, PHLDB1, RNU6-1157P, } \\
\quad \text { RNU6-376P, TREH, TREHP) } \\
\text { PBC 22:39670851-39747780 (CACNA1I, ENTHD1) }\end{array}$ \\
\hline SZ-UC & $\begin{array}{l}\text { 8/205 } \\
\text { UC 11:63804569-65898631** (CCDC88B, RPS6KA4, TRPT1, FLRT1) }\end{array}$ \\
\hline Tourette's syndrome-allergy & $0 / 0$ \\
\hline
\end{tabular}

Note. This table summarizes findings of local genetic correlation analysis, including the number of significantly correlated loci before and after BenjaminiHochberg $(\mathrm{BH})$ correction for multiple testing (shown in bold). Loci that showed robust correlations were interrogated for co-localization with significant genome-wide associations (GWS hits, with $p<5 \times 10^{-8}$ ). The chromosomal coordinates containing GWS signal are provided, along with associated genes. Proposed candidate genes are highlighted with bold text. $\mathrm{ADHD}=$ attention deficit-hyperactivity disorder; $\mathrm{BD}=$ bipolar disorder; $\mathrm{BH}=\mathrm{Benjamini}-\mathrm{Hoch}-$ berg; $\mathrm{CD}=$ Crohn's disease; $\mathrm{CEA}=$ childhood ear infection; $\mathrm{HPT}=$ hypothyroidism; $\mathrm{NC}-\mathrm{H}=$ comparison; $\mathrm{OCD}=$ obsessive compulsive disorder; $\mathrm{PBC}=$ primary biliary cirrhosis; RA = rheumatoid arthritis; SLE = systemic lupus erythematosus; SZ = schizophrenia; UC = ulcerative colitis.

causality and temporality of these relationships is not clearly established (Benros, 2013; Cucino \& Sonnenberg, 2001; Dickerson et al., 2011; Eaton, Pedersen, Nielsen, \& Mortensen, 2010; Marrie et al., 2017a, Marrie et al., 2017b; Sidhom et al., 2012). Positive genetic inter-correlations among these phenotypes are also consistent with recent work demonstrating that the positive correlation between $\mathrm{BD}$ and SZ are significantly mediated by both CNS and immunologic tissues (Lu et al., 2017). Our local genetic correlation analyses were inadequately powered to detect loci relevant to most of the psychiatricimmune disorder pairs, including BD. However, comparisons with SZ yielded 97 loci that were robust to multiple test correction, 18 of which also were shown to harbor GW hits in previous studies. In several instances, these GW hits localize near genes with functions that are pleiotropic and relevant to both brain and immune system phenotypes. For example, we identified a SZ-CD correlated locus at $4 q 24$ (4:100678360-103221356) that contained GW hits for both SZ (putatively attributed to SLC39A8) and several autoimmune diseases (putatively attributed to NFKB1 and MANBA); others have proposed that associations at this locus may exert pleiotropic effects on a wide range of phenotypes (additionally including body mass index, serum levels of 


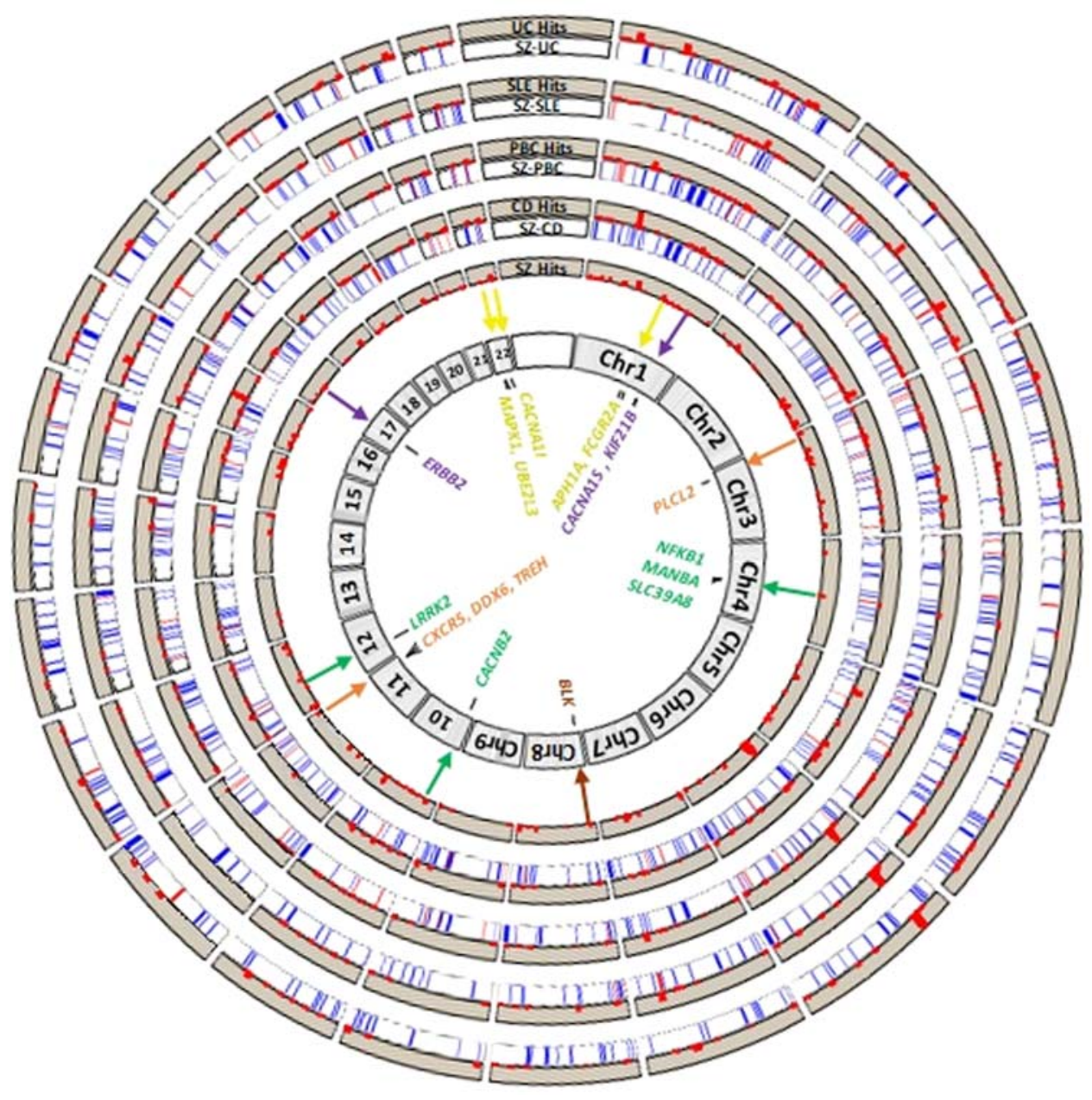

FIGURE 2 This figure depicts the HESS local genetic correlation data with respect to the genome and previously reported genome-wide association signals for respective disorders. A model genome using HG19 coordinates is depicted in gray. Moving outward from the center of the plot, the first track containing a red histogram depicts loci significantly associated with SZ (GWAS $p<5 \times 10^{-8}$ ), with larger peaks indicating more significance (plotted as - $\log (p$-value)). The second track (labeled SZ-CD) depicts regions of genetic correlation between SZ and CD, such that blue reflects uncorrected $p<.05$ and red reflects BH-corrected $p<.05$. The next track (labeled CD Hits) contains a histogram depicting CD GWAS signal as described previously. The next pair of tracks depict genetic correlations for SZ-PBC and PBC GWAS signal, respectively. The third pair of tracks depicts this information for SZ-SLE (with SLE GWAS signal). The fourth pair of tracks depicts this information for SZ-UC and UC GWAS signal, respectively. In the center of the plot, we identify several GWAS candidate genes using colored text and arrows to indicate the pertinent locus; colored text and arrows are used to indicate the relevant phenotype-pairs, such that green $=\mathrm{SZ}-\mathrm{CD}$, orange $=\mathrm{SZ}-\mathrm{PBC}$, yellow $=$ SZ-SLE, brown $=$ SZ-PBC/SLE, and purple $=$ SZ/BD-CD/PBC/UC [Color figure can be viewed at wileyonlinelibrary.com]

manganese, $\mathrm{N}$-terminal pro-B-type natriuretic peptide, and HDL-cholesterol) through a functional variant found in European populations affecting the SLC39A8 cation transporter (Costas, 2017; Li et al., 2016). A locus within 11q23.3 (11:117747110-119215476) was significantly correlated between SZ and PBC and harbors a region of GW hits for multiple autoimmune disorders attributed to PLCL2, a catalytically inactive phospholipase-like protein thought to influence intracellular signaling, calcium homeostasis, and GABA-ergic receptor trafficking in immune and neuronal cell types, among others (Murakami, Matsuda, Harada, \& Hirata, 2017; Takenaka et al., 2003; Toyoda et al., 2015). A de novo missense mutation affecting this gene was identified in an exome sequencing study of SZ affected individuals, though no replication appears to have been reported ( $\mathrm{Xu}$ et al., 2011). Similarly, a correlated locus within 22q13.1 (22:39307894-40545797, highlighted yellow in Figure 2) contains GW hits for PBC, which overlaps with voltage-gated calcium channel gene CACNA1l; this gene has been implicated by both GWAS and rare-variant studies of SZ (Andrade et al., 2016; Ripke, 2014). Another correlated locus within 11q23 (11:118579747-118743772) contained GW hits for multiple autoimmune disorders and is suspected to exert pleiotropic effects through several genes, whose functions include repression of aberrant interferon signaling (DDX6; Lumb et al., 2017), chemokine signaling between T-helper and B-cells (CXCR5; Papp, Szabó, Szekanecz, \& Zeher, 2014; Vaeth et al., 2014), and enzymatic break down of microbial disaccharides (TREH; Muller et al., 2013). Notably, functional genomic studies have identified DDX6 as a gene that is perturbed during neuronal differentiation of samples derived from individuals with schizophrenia (Maschietto et al., 2015), and as a peripheral blood marker of cerebrospinal fluid serotonin metabolite levels (Luykx et al., 2016), supporting its relevance to psychiatric phenotypes.

We also examined loci that showed a nominal genetic correlation across multiple disorder pairs, and found these loci also harbored GW 
hits for respective phenotypes. The locus at $17 q 12$ shared among multiple disorders contains a GW hit for BD (17:3680934438877404) ascribed to candidate gene ERBB2 (Hou et al., 2016). This gene and its relatives encode receptor tyrosine kinases that interact with a family of growth factors called neuregulins to regulate the assembly of neural circuitry, myelination, neurotransmission and synaptic plasticity. A large body of evidence implicates both ligands and receptors from these families as susceptibility genes for SZ and BD (Mei \& Nave, 2014). Notably, ERBB2 overlaps with GW hits for multiple autoimmune disorders, though these have been attributed to different genes in the region. Another locus at 1q32.1 (1:200137649-201589975) contains GW hits for multiple autoimmune disorders (including celiac disease, CD, multiple sclerosis, and UC) and is near candidate genes C1orf106, CACNA1S, GPR25, and KIF21B. Genetic disruptions of voltage-gated calcium channels, including CACNA1S, are well-established susceptibility factors in psychiatric and neurological disorders (Heyes et al., 2015; Schmunk \& Gargus, 2013). KIF21B encodes a neuronal motor protein implicated in $\mathrm{GABA}_{\mathrm{A}}$ receptor trafficking (Labonte, Thies, \& Kneussel, 2014), in addition to having a suspected role in regulating inflammatory signaling in several lymphocyte subtypes (Goris, Boonen, D'hooghe, \& Dubois, 2010).

While it is tempting to speculate about these observations, we must acknowledge limitations and caveats of the present approach. Current methods for assessing genetic correlations are not well suited for fine-mapping shared liability across disorders; other methods are better suited for this task, including extensions of GWAS that model multiple phenotypes simultaneously (Cotsapas, 2011; Porter \& O'Reilly, 2017; Turley et al., 2018; Wang et al., 2015). With respect to local genetic correlations, we have prioritized reporting of loci that co-localize with GW hits. However, this implies that the presence of the GW hit is contributing to the observed correlation, which we have not demonstrated presently. As such, our discussion of potentially pleiotropic loci and candidate genes should be considered anecdotal at this time. One indirect approach to assessing the role of GW hits in a local genetic correlation might be to reestimate the local correlation after the removal of the smaller region of GW signal from the original datasets. When we conducted this analysis for the SZ-CD pair, we found that the number of significant loci ( $\mathrm{BH} p<0.05)$ was reduced from 32 to 8 , suggesting that $\mathrm{GW}$ hits likely play an important role in many of the local genetic correlations. Future studies will be able to combine larger GWAS sample sizes with new methods aimed at stratifying genetic correlations by biological annotations (e.g., tissue type or signaling pathways) in order to more precisely define the parts of the genome that mediate a genetic correlation (Lu et al., 2017).

Several methods have now been used to examine quantitative SNP-based genetic relationships between psychiatric and immunerelated phenotypes, including restricted maximum likelihood (REML) co-heritability, polygenic risk scores, genetic analysis incorporating pleiotropy and annotations, and other permutation-based methods (Cross-Disorder Group of the Psychiatric Genomics Consortium, 2013; Lee et al., 2013; Pouget et al., 2016; Wang et al., 2015). Different approaches rest on unique assumptions, test different sets of hypotheses, and appear prone to generating sometimes conflicting 
TABLE 5 HESS analysis of putative causal directionality

\begin{tabular}{llll}
$\begin{array}{l}\text { Phenotype 1, } \\
\text { phenotype 2 }\end{array}$ & $\begin{array}{l}\text { Local Genetic correlation } \pm \text { error } \\
\text { at loci reaching GWS only } \\
\text { for phenotype 1 }\end{array}$ & $\begin{array}{l}\text { Local genetic correlation } \pm \text { error } \\
\text { at loci reaching GWS only } \\
\text { for phenotype 2 }\end{array}$ & $\begin{array}{l}\text { Suggested direction } \\
\text { SZ-CD }\end{array}$ \\
\hline $0.37 \pm 0.09$ & $0.11 \pm 0.08$ & $\mathrm{SZ} \rightarrow \mathrm{CD}$ \\
SZ-PBCS & $0.58 \pm 0.18$ & $0.26 \pm 0.17$ & $\mathrm{SZ} \rightarrow \mathrm{PBC}$ \\
\hline SZ-SLE & $0.26 \pm 0.13$ & $0.16 \pm 0.16$ & $\mathrm{SZ} \rightarrow \mathrm{SLE}$ \\
\hline SZ-UC & $0.43 \pm 0.09$ & $0.16 \pm 0.10$ & $\mathrm{SZ} \rightarrow \mathrm{UC}$ \\
\hline
\end{tabular}

Note. Depicts the results of HESS analysis of putative causal directionality. Within this analysis, local genetic correlations are examined within loci containing GWS associations for each phenotype. The phenotype for which GWS loci produce the larger local correlations suggests that genetic liability for this phenotype may contribute to genetic risk for the other, especially when the correlation error bounds of the second phenotype overlap with zero. When both phenotypes show correlations overlapping with zero, no directionality is supported. $C D=$ Crohn's disease; GWS = genome-wide significance defined as $p<5 \times 10^{-8} ; \mathrm{PBC}=$ primary biliary cirrhosis; $\mathrm{SLE}=$ systemic lupus erythematosus; $\mathrm{UC}=$ ulcerative colitis.

results. Using several approaches that were not dependent on the directionality of a given SNP's effect, Wang and colleagues concluded that many (24 of 35 ) pairs of psychiatric and immune-related phenotypes shared a statistically significant proportion of risk-associated loci; among these findings was a significant genetic overlap between BD (as well as SZ) and UC (Wang et al., 2015). However, many of the other relationships identified in that study were not significant in the present study. Another recent study demonstrated that polygenic risk scores reflecting additive risk for several autoimmune diseases can explain a small proportion of variance in SZ case-control status, yet the genome-wide significant SNPs from the autoimmune GWASs were not over-represented among SZ's genome-wide significant hits when permutation-based analysis was performed (Pouget et al., 2016). The apparent disagreement between different approaches for assessing shared genetic liability thus underscores the value of examining the consensus across studies and methods (Pouget et al., 2016).

The LDSC approach featured here attempts to quantitate similarities and differences in association signals across the entire genome. Some of our phenotype-pairs have been examined previously using genome-wide assessment methods, yielding apparently contradictory findings (Anttila, 2016; Bulik-Sullivan, Loh, et al., 2015b; Zheng et al., 2016). For example, a previous study implementing a REML-based approach did not find significant SNP-based co-heritabilities between $C D$ and the major psychiatric phenotypes (Lee et al., 2013). Additionally, the first study implementing the LDSC method found no significant correlation ( $r g=0.08 \pm 0.08$, uncorrected $p=0.33$ ) between BD and UC (Bulik-Sullivan, Finucane, et al., 2015a); this study used a smaller dataset for BD (Sklar et al., 2011; $N=16,731$ ) and a different version of the UC dataset (reported as Jostins et al., 2012; $N=$ 27,432). A similar non-correlation is also reported in LD-Hub (http:// Idsc.broadinstitute.org/), using what appears to be the same datasets, although referencing a related article (Liu et al., 2015; $N=27,432$ ). The analyses portrayed in our main text utilized a larger BD dataset (Hou et al., 2016; $N=40,225$ ), the same dataset for UC (Liu et al., 2015; $N=27,432$ ), and uniform criteria for SNP retention based on inclusion in the HapMap3 panel and MAF $\geq 5 \%$ within the 1000 Genomes Project Phase 3 European samples. In order to resolve apparent discrepancies, we obtained additional versions of the available data for BD, SZ, CD, and UC and pre-filtered under both inclusive (imputation INFO score $\geq 0.9$ or all SNPs, when INFO score unavailable) or exclusive criteria (MAF > 5\% within the 1000 Genomes Project Phase 3 European samples). We found that correlations between $\mathrm{SZ}$ and each of $\mathrm{CD}, \mathrm{PBC}$, and $\mathrm{UC}$ tended to be more positive and more significant (i.e., reaching a $\mathrm{BH}$-corrected threshold) when using the SZ data filtered at MAF > 5\% (Supporting Information Figure S3). A similar pattern held true for inclusive vs. exclusive pre-filtering for the BD dataset generated by Sklar et al., but this was not the case for the larger Hou et al., dataset. A side-byside comparison of the effects of different pre-filtering decisions for the $\mathrm{BD}, \mathrm{SZ}, \mathrm{CD}$, and $\mathrm{UC}$ datasets in relation to the other phenotypes is provided in Supporting Information Figure S4. These observations indicate that decisions pertaining to SNP inclusion can have a considerable effect on the result of the LDSC analysis; this idea is further supported by the observation that stratified genetic correlation analyses based on MAF thresholds can produce different levels of statistical significance and opposite patterns of correlation directionality (Lu et al., 2017). Thus, our study suggests that genetic correlations between psychiatric and immune-related disorders may be more significant when analyses are restricted to common variation. Reassuringly, the developers of the HESS method use the same datasets examined presently, and also report positive genetic correlations between SZ and the inflammatory bowel disorders (Shi et al., 2017). The results of the HESS analysis of putative causal directionality indicate that the local genetic correlations are higher in loci occupied by SZ GW hits, as compared to the loci harboring hits for the paired autoimmune disorders (Shi et al., 2017). This pattern is consistent with the hypothesis that genetic liability toward SZ tends to impart a greater genetic risk for the corresponding paired disorder, rather than the opposite directional hypothesis. A related interpretation may be there is an unobserved intermediate phenotype (e.g., a shared biological pathways/mechanism) that is pleiotropic for both measured phenotypes, but more strongly influences the SZ phenotype. This pattern of findings could also be caused by the presence of a confounding factor (e.g., smoking, socioeconomic status) that portends risk for both phenotypes (Shi et al., 2017). Thus, we caution against overinterpretation of these findings. Extensions of Mendelian randomization methods to incorporate two GWAS samples using multi-allelic risk stratifying instruments will be better suited to address these hypotheses (Hartwig, Davies, Hemani, \& Davey Smith, 2016), especially as future GWASs provide well-powered genetic estimates of potentially relevant intermediate phenotypes (e.g., brain structure morphometry, circulating immune cell phenotypes, and serum cytokine levels (Ahola-Olli, 2017; Astle et al., 2016; Hibar et al., 2015). Other limitations of the HESS method, including assumptions related 
to sample overlap and ancestry stratification, are discussed extensively by the method's developers (Shi et al., 2017).

Our study also identified many phenotype-pairs that demonstrated significant genome-wide correlations using the LDSC method, but for which HESS-based genome-wide and local genetic correlations could not be identified. This is unsurprising, given that the sample sizes for these phenotypes were generally below the recommended sample size for HESS analyses ( $N \geq 50,000$; Shi et al., 2017) Nonetheless, some of these relationships are supported by evidence from clinical and epidemiological studies, and thus may warrant follow-up using larger sample sizes and alternative methods for assessing genetic relationships. For example, we observed a modest positive correlation between self-reported hypothyroidism and major depression $\left(r g=0.33 \pm 0.09, p=5.0 \times 10^{-4}\right)$, as well as trait neuroticism $\left(r g=0.25 \pm 0.06, p=7.2 \times 10^{-5}\right)$. This could be consistent with two different sets of clinical observations. The first is that symptoms of depression are common in individuals with hypothyroidism, and that subclinical hypothyroidism could play a role in a subset of persons diagnosed with major depression; thus cross-contamination of GWAS samples could lead to a biased positive correlation. However, the second observation is that there is an increased incidence of major depression and depressive symptomatology in persons with autoimmune thyroiditis receiving hormone replacement therapy (Dayan \& Panicker, 2013; Giynas Ayhan, Uguz, Askin, \& Gonen, 2014). It is worth noting that GWAS data for allergy, asthma, hypothyroidism, childhood ear infection, and Parkinson's disease were obtained through 23andMe, Inc. These data are based on self-report, and thus could be more susceptible to bias stemming from misdiagnosis or misreporting, though previous work supports their validity (Tung et al., 2011). None the less, the samples sizes are an order of magnitude larger than many other datasets, resulting in smaller standardized errors and better power for the detection of weak genetic correlations. It is yet unclear whether small magnitude genetic correlations like these might be clinically meaningful. The LDSC correlations observed presently were relatively weak magnitude $(r g s \approx 0.12$ to 0.30$)$ and of modest statistical significance $\left(1 \times 10^{-5} \leq\right.$ uncorrected $p \leq 5 \times 10^{-3}$ ), when compared to the strongest genetic correlations observed within each group of datasets (e.g., SZ-BD $r g=0.87$ with $p=7.4 \times 10^{-94} ; \mathrm{CD}-\mathrm{UC}$ rg $=0.71$ with $\left.p=3.5 \times 10^{-36}\right)$.

Several other significant genetic correlations are supported in the clinical and epidemiological literature. For example, we found a positive correlation between ADHD and rheumatoid arthritis ( $r g=$ $0.16 \pm 0.05, p=9.0 \times 10^{-4}$ ); this finds support in large registry-based studies indicating an increase in ADHD diagnosis in individuals with autoimmune disease (Nielsen, Benros, \& Dalsgaard, 2017), children with mother's affected by autoimmune disease (Nielsen et al., 2017), and children of mothers with rheumatoid arthritis (Instanes et al., 2017). Registry-based studies also provide support for increased incidence of ear infections $\left(r g=0.20 \pm 0.05, p=2.0 \times 10^{-4}\right)$ and psoriasis $\left(r g=0.23 \pm 0.07, p=1.0 \times 10^{-3}\right)$ among individuals with ADHD (Adesman, 1990; Hegvik, Instanes, Haavik, Klungsøyr, \& Engeland, 2017; Nielsen et al., 2017; Silva, Colvin, Hagemann, Stanley, \& Bower, 2014). On the other hand, ADHD was positively correlated with CRP $\left(r g=0.23 \pm 0.06, p=2.0 \times 10^{-4}\right)$, though a relatively large epidemiological study finds no association in affected individuals
(Vogel et al., 2017). The negative correlation between anorexia nervosa and CRP $\left(r g=-0.30 \pm 0.08, p=1.0 \times 10^{-4}\right)$ is borne out in a recent meta-analysis of relevant studies (Solmi et al., 2015). Another negative correlation between OCD and type 1 diabetes $(r g=-0.32 \pm$ $0.11, p=5.4 \times 10^{-3}$ ) finds no support within a limited body of literature (Sivertsen, Petrie, Wilhelmsen-Langeland, \& Hysing, 2014). However, the positive correlation between Tourette syndrome and allergy $\left(r g=0.24 \pm 0.06, p=2.7 \times 10^{-5}\right)$ is consistent with evidence of increased comorbidity between these phenotypes (Chang, 2011; Yuce et al., 2014). There is a paucity of clinical studies directly assessing the relationship between SZ and PBC $(r g=0.14 \pm 0.05, p=2.0$ $\left.\times 10^{-3}\right)$. On the other hand, the correlation between SZ and SLE ( $r g=$ $0.15 \pm 0.04, p=2.0 \times 10^{-4}$ ) appears to be supported by both epidemiological evidence of increased comorbidity (Tiosano et al., 2016) and the well-documented (although rare) phenomenon of CNS lupus presenting with SZ-like symptoms (Pego-Reigosa \& Isenberg, 2008), which may contribute to misdiagnosis. Finally, positive correlations involving cigarette smoking behavior and CRP $(r g=0.31 \pm 0.07, p=$ $\left.3.6 \times 10^{-5}\right)$, as well as rheumatoid arthritis $(r g=0.17 \pm 0.05, p=2.3$ $\times 10^{-3}$ ), are perhaps unsurprising given considerable evidence of elevated CRP in persons who smoke (Ohsawa et al., 2005), and increased incidence of smoking behavior among individuals diagnosed with rheumatoid arthritis (Di Giuseppe, Discacciati, Orsini, \& Wolk, 2014). These findings may indicate a need for more adequate statistical treatment of smoking behavior in GWAS studies.

The present study identified a number of intriguing and previously unreported genetic correlations, some of which appear to localize near established risk factors for complex disease. On the whole, these findings are consistent with the idea that similar signatures of common genetic variation may increase risk for both psychiatric and immune-related disorders. However, it is important to keep in mind that these findings do not necessarily imply causality or even shared genetic etiology. SNP-based genetic correlations could arise from a wide variety of underlying factors, including the possibility that the relationship between phenotypes is mediated by behavioral or cultural factors, or influenced by a heritable but unexamined underlying trait that confers risk to both phenotypes (Anttila, 2016; Bulik-Sullivan, Finucane, et al., 2015a). Other factors that could contribute to genetic correlations include effects mediated by parental genotypes and their influence on parental behaviors that impact the offspring (Coop \& Pickrell, 2016). Additionally, GWAS studies of psychiatric phenotypes typically do not screen affected cases on the presence of other medical conditions (and vice versa), thus over-representation of a given phenotype in the sample of another phenotype could bias the data toward the detection of a genetic correlation. Finally, estimates of genetic similarities could be influenced by misdiagnosed cases (Wray, Lee, \& Kendler, 2012). Other general limitations of this method (in comparison with other approaches) have been discussed previously elsewhere (Anttila, 2016; Bulik-Sullivan, Finucane, et al., 2015a). In light of the exploratory nature of the present study, another critique pertains to the lack of clearly identified positive and negative control comparisons. Additionally, the clinical significance of weak or modest genetic correlations is yet unclear. Future work could shed light on this topic by comparing the strength of reported genetic correlations with estimates of effect size from 
epidemiological associations, in order to create an atlas of concordance and shed light on the sensitivity and specificity of these genetic methods. One final critique of this approach is that it falls short of identifying plausible genetic and biological mechanisms that mediate potentially pleiotropic loci. Future work incorporating expression quantitative trait loci, differentially expressed or methylated genes, or enriched ontological and functional terms may provide a clearer context for assessing biological similarities between phenotypes. Despite these limitations, the present study indicates that shared aspects of common genetic variation may underlie longrecognized epidemiological links between psychiatric and immunerelated disorders and serves as a start point for the identification and characterization of potentially pleiotropic loci.

\section{ACKNOWLEDGMENTS}

The authors gratefully acknowledge the contributions of all the individuals (patients, families, research participants, clinicians and diagnosticians, research associates, and data analysts) and consortia whose efforts made possible the GWAS studies and meta-analyses featured in the present study. For most of the phenotypes examined in the present study, clinical and genetic data were collected across numerous sites, each with their own unique patients, staff, and funding sources. While we attempted to provide more thorough recognition of the required acknowledgments for each individual phenotype in our supplementary note, we realize that it is not possible to recognize every individual and funding mechanism that made these studies possible, and we apologize for this. We gratefully acknowledge 23andMe, Inc., its staff, and its customers who consented to participate in research. We also gratefully acknowledge the developers of the LDSC and HESS software. We gratefully acknowledge Susan Service for her assistance preparing and analyzing the data supporting the original association studies of neurocognitive impairment and dementia in HIV-affected adults.

\section{CONFLICT OF INTEREST}

The authors declare no conflicts of interest related to this study.

\section{ORCID}

Daniel S. Tylee (D) http://orcid.org/0000-0002-7579-6096

Bradford B. Worrall (D) http://orcid.org/0000-0001-9386-4091

\section{REFERENCES}

Adesman, A. R. (1990). Otitis media in children with learning disabilities and in children with attention deficit disorder with hyperactivity. Pediatrics, 85(3), Retrieved from http://pediatrics.aappublications.org/ content/85/3/442

Ahola-Olli, A. V. (2017). Genome-wide association study identifies 27 loci influencing concentrations of circulating cytokines and growth factors. American Journal of Human Genetics, 100(1), 40-50. Retrieved from http://www.ncbi.nlm.nih.gov/pubmed/27989323

Anderson, C. A. (2011). Meta-analysis identifies 29 additional ulcerative colitis risk loci, increasing the number of confirmed associations to 47 . Nature Genetics, 43(3), 246-252. Retrieved from http://www. pubmedcentral.nih.gov/articlerender.fcgi?artid=3084597\&tool= pmcentrez\&rendertype=abstract.
Andrade, A., Hope, J., Allen, A., Yorgan, V., Lipscombe, D., \& Pan, J. Q. (2016). A rare schizophrenia risk variant of CACNA1I disrupts CaV3.3 channel activity. Scientific Reports, 6(1), 34233. Retrieved from http:// www.nature.com/articles/srep34233

Anney, R. J. L. (2017). Meta-analysis of GWAS of over 16,000 individuals with autism spectrum disorder highlights a novel locus at 10q24.32 and a significant overlap with schizophrenia. Molecular Autism, 8(1).

Anttila, V. (2016). Analysis of shared heritability in common disorders of the brain. Cold Spring Harbor Labs Journals (bioRvix.org).

Arnold, P. D. (2017). Revealing the complex genetic architecture of obsessive-compulsive disorder using meta-analysis. Molecular Psychiatry, Retrieved from http://www.ncbi.nlm.nih.gov/pubmed/28761083

Astle, W. J., Elding, H., Jiang, T., Allen, D., Ruklisa, D., Mann, A. L., ... Soranzo, N. (2016). The allelic landscape of human blood cell trait variation and links to common complex disease. Cell, 167(5), 1415-1429. e19. Retrieved from http://www.ncbi.nlm.nih.gov/pubmed/27863252

Benros, M. E. (2013). Autoimmune diseases and severe infections as risk factors for mood disorders: A nationwide study. JAMA Psychiatry, 70(8), 812-820. Retrieved from http://archpsyc.jamanetwork.com. libproxy1.upstate.edu/article.aspx?articleid=1696348

Bentham, J., Morris, D. L., Cunninghame Graham, D. S., Pinder, C. L., Tombleson, P., Behrens, T. W., ... Vyse, T. J. (2015). Genetic association analyses implicate aberrant regulation of innate and adaptive immunity genes in the pathogenesis of systemic lupus erythematosus. Nature Genetics, 47(12), 1457-1464. Retrieved from http://www. nature.com/doifinder/10.1038/ng.3434.

Berisa, T., \& Pickrell, J. K. (2015). Approximately independent linkage disequilibrium blocks in human populations. Bioinformatics, 32(2), 283-285.

Bolton, J. L. (2014). Genome wide association identifies common variants at the SERPINA6/SERPINA1 locus influencing plasma cortisol and corticosteroid binding globulin. PLoS Genetics, 10(7), e1004474. Retrieved from http://www.pubmedcentral.nih.gov/articlerender.fcgi?artid= 4091794\&tool=pmcentrez\&rendertype=abstract

Bradfield, J. P., Qu, H.-Q., Wang, K., Zhang, H., Sleiman, P. M., Kim, C. E., ... Hakonarson, H. (2011). A genome-wide meta-analysis of six type 1 diabetes cohorts identifies multiple associated loci. PLoS Genetics, 7(9), e1002293.

Bulik-Sullivan, B. K., Finucane, H. K., Anttila, V., Gusev, A., Day, F. R., Loh, P.-R., ... Neale, B. M. (2015a). An atlas of genetic correlations across human diseases and traits. Nature Genetics, 47(11), 1236-1241. Retrieved from http://www.ncbi.nlm.nih.gov/pubmed/26414676

Bulik-Sullivan, B. K., Loh, P.-R., Finucane, H. K., Ripke, S., Yang, J., Patterson, N., ... Neale, B. M. (2015b). LD Score regression distinguishes confounding from polygenicity in genome-wide association studies. Nature Genetics, 47(3), 291-295. Retrieved from https://doi. org/10.1038/ng.3211

Chang, Y. T. (2011). Correlation of Tourette syndrome and allergic disease: Nationwide population-based case-control study. Journal of Developmental and Behavioral Pediatrics, 32(2), 98-102.

Coop, G., \& Pickrell, J. (2016). What is genetic correlation? joepickrell.wordpress.com/. Retrieved from https://joepickrell.wordpress. com/2016/04/19/

what-is-genetic-correlation/

Cordell, H. J. (2015). International genome-wide meta-analysis identifies new primary biliary cirrhosis risk loci and targetable pathogenic pathways. Nature Communications, 6, 8019. Retrieved from http://www. nature.com/ncomms/2015/150922/ncomms9019/full/ncomms9019. html\%5Cnhttp://www.pubmedcentral.nih.gov/articlerender.fcgi? artid $=4580981 \&$ tool $=$ pmcentrez $\&$ rendertype $=$ abstract $\%$ 5Cnhttp://www.nature.com/doifinder/10.1038/ncomms9019.

Costas, J. (2017). The highly pleiotropic gene SLC39A8 as an opportunity to gain insight into the molecular pathogenesis of schizophrenia. American Journal of Medical Genetics Part B: Neuropsychiatric Genetics, Retrieved from http://www.ncbi.nlm.nih.gov/pubmed/28557351

Cotsapas, C. (2011). Pervasive sharing of genetic effects in autoimmune disease. PLoS Genetics, 7(8), e1002254. Retrieved from http://www. scopus.com/inward/record.url?eid=2-s2.0-80052325959\& partnerID=tZOtx3y1

Cross-Disorder Group of the Psychiatric Genomics Consortium. (2013). Identification of risk loci with shared effects on five major psychiatric 
disorders: A genome-wide analysis. The Lancet, 381(9875), 13711379.

Cucino, C., \& Sonnenberg, A. (2001). The comorbid occurrence of other diagnoses in patients with ulcerative colitis and Crohn's disease. The American Journal of Gastroenterology, 96(7), 2107-2112.

Curtis, J., Luo, Y., Zenner, H. L., Cuchet-Lourenço, D., Wu, C., Lo, K., ... Nejentsev, S. (2015). Susceptibility to tuberculosis is associated with variants in the ASAP1 gene encoding a regulator of dendritic cell migration. Nature Genetics, 47(5), 523-527. Retrieved from https:// doi.org/10.1038/ng.3248\%5Cnhttp://www.ncbi.nlm.nih.gov/ pubmed/25774636\%5Cnhttp://www.pubmedcentral.nih.gov/ articlerender.fcgi?artid=PMC4414475.

Dayan, C. M., \& Panicker, V. (2013). Hypothyroidism and depression. European Thyroid Journal, 2(3), 168-179. Retrieved from http://www. ncbi.nlm.nih.gov/pubmed/24847450

Dehghan, A. (2011). Meta-analysis of genome-wide association studies in \&gt;80 000 subjects identifies multiple loci for C-reactive protein levels. Circulation, 123(7), 731-738. Retrieved from http://www.ncbi. nlm.nih.gov/pubmed/21300955\%5Cnhttp://www.pubmedcentral. nih.gov/articlerender.fcgi?artid=PMC3147232.

Demontis, D. (2017). Discovery of the first genome-wide significant risk loci For ADHD. bioRxiv, 145581. Retrieved from https://www.biorxiv. org/content/early/2017/06/03/145581

de Moor, M. H. M., Costa, P. T., Terracciano, A., Krueger, R. F., de Geus, E. J., Toshiko, T., ... Boomsma, D. I. (2012). Meta-analysis of genome-wide association studies for personality. Molecular Psychiatry, 17(3), 337-349. Retrieved from http://www.pubmedcentral.nih.gov/articlerender.fcgi? artid $=3785122 \&$ tool $=$ pmcentrez\&rendertype $=$ abstract

Deverman, B. E., \& Patterson, P. H. (2009). Cytokines and CNS development. Neuron, 64(1), 61-78. Retrieved from http://www.ncbi.nlm.nih. gov/pubmed/19840550

Dickerson, F., Stallings, C., Origoni, A., Vaughan, C., Khushalani, S., Alaedini, A., \& Yolken, R. (2011). Markers of gluten sensitivity and celiac disease in bipolar disorder. Bipolar Disorders, 13(1), 52-58. Retrieved from http://www.ncbi.nlm.nih.gov/pubmed/21320252

Di Giuseppe, D., Discacciati, A., Orsini, N., \& Wolk, A. (2014). Cigarette smoking and risk of rheumatoid arthritis: A dose-response meta-analysis. Arthritis Research \& Therapy, 16(2), R61. Retrieved from http://arthritis-research.biomedcentral.com/articles/10.1186/ar4498

Dowlati, Y. (2010). A meta-analysis of cytokines in major depression. Biological Psychiatry, 67(5), 446-457. Retrieved from http://www.ncbi. nlm.nih.gov/pubmed/20015486

Dubois, P. C. A. (2010). Multiple common variants for celiac disease influencing immune gene expression. Nature Genetics, 42(4), 295-302. Retrieved from http://www.pubmedcentral.nih.gov/articlerender.fcgi? artid $=2847618 \&$ tool $=$ pmcentrez\&rendertype $=$ abstract.

Duncan, L. E. (2017a). Largest GWAS of PTSD ( $N=20$ 070) yields genetic overlap with schizophrenia and sex differences in heritability. Molecular Psychiatry, 1-8.

Duncan, L. E., Yilmaz, Z., Gaspar, H., Walters, R., Goldstein, J., Anttila, V., ... Bulik, C. M. (2017b). Significant locus and metabolic genetic correlations revealed in genome-wide association study of anorexia nervosa. American Journal of Psychiatry, 174(9), 850-858. Retrieved from http://ajp.psychiatryonline.org/doi/10.1176/appi.ajp.2017.16121402

EArly Genetics and Lifecourse Epidemiology (EAGLE) Eczema Consortium, Australian Asthma Genetics Consortium (AAGC), \& Australian Asthma Genetics Consortium (AAGC). (2015). Multi-ancestry genome-wide association study of 21,000 cases and 95,000 controls identifies new risk loci for atopic dermatitis. Nature Genetics, 47(12), 1449-1456. Retrieved from http://www.ncbi.nlm.nih.gov/pubmed/26482879.

Eaton, W. W., Byrne, M., Ewald, H., Mors, O., Chen, C.-Y., Agerbo, E., \& Mortensen, P. B. (2006). Association of schizophrenia and autoimmune diseases: Linkage of Danish national registers. American Journal of Psychiatry, 163(3), 521-528. Retrieved from http://www.ncbi.nlm. nih.gov/pubmed/16513876

Eaton, W. W., Pedersen, M. G., Nielsen, P. R., \& Mortensen, P. B. (2010). Autoimmune diseases, bipolar disorder, and non-affective psychosis. Bipolar Disorders, 12(6), 638-646. Retrieved from http://www. pubmedcentral.nih.gov/articlerender.fcgi?artid=2950824\&tool= pmcentrez\&rendertype=abstract
Edwards, A. C., Aliev, F., Wolen, A. R., Salvatore, J. E., Gardner, C. O., McMahon, G., ... Kendler, K. S. (2015). Genomic influences on alcohol problems in a population-based sample of young adults. Addiction (Abingdon, England), 110(3), 461-470. Retrieved from http:// www.pubmedcentral.nih.gov/articlerender.fcgi?artid $=4329073 \&$ tool=pmcentrez\&rendertype $=$ abstract

Eicher, J. D., Powers, N. R., Miller, L. L., Akshoomoff, N., Amaral, D. G., Bloss, C. S., ... Gruen, J. R. (2013). Genome-wide association study of shared components of reading disability and language impairment. Genes, Brain, and Behavior, 12(8), 792-801. Retrieved from http:// www.pubmedcentral.nih.gov/articlerender.fcgi?artid=3904347\& tool=pmcentrez\&rendertype $=$ abstract

Ellinghaus, E., Stuart, P. E., Ellinghaus, D., Nair, R. P., Debrus, S., Raelson, J. V., ... Franke, A. (2012). Genome-wide meta-analysis of psoriatic arthritis identifies susceptibility locus at REL. Journal of Investigative Dermatology, 132(4), 1133-1140.

Felger, J. C., \& Lotrich, F. E. (2013). Inflammatory cytokines in depression: Neurobiological mechanisms and therapeutic implications. Neuroscience, 246, 199-229. Retrieved from http://www.pubmedcentral.nih. gov/articlerender.fcgi?artid=3741070\&tool=pmcentrez\& rendertype $=$ abstract

Fineberg, A. M., \& Ellman, L. M. (2013). Inflammatory cytokines and neurological and neurocognitive alterations in the course of schizophrenia. Biological Psychiatry, 73(10), 951-966. Retrieved from http://www. scopus.com/inward/record.url?eid=2-s2.0-84876945142\& partnerID=tZOtx3y1

Fischer, A., Schmid, B., Ellinghaus, D., Nothnagel, M., Gaede, K. I., Schürmann, M., ... Schreiber, S. (2012). A novel sarcoidosis risk locus for Europeans on chromosome 11q13.1. American Journal of Respiratory and Critical Care Medicine, 186(9), 877-885.

Franke, A., McGovern, D. P., Barrett, J. C., Wang, K., Radford-Smith, G. L., Ahmad, T., ... Parkes, M. (2010). Genome-wide meta-analysis increases to 71 the number of confirmed Crohn's disease susceptibility loci. Nature Genetics, 42(12), 1118-1125. Retrieved from http://www.pubmedcentral. nih.gov/articlerender.fcgi?artid=3299551\&tool=pmcentrez\& rendertype $=$ abstract.

Gelernter, J., Kranzler, H. R., Sherva, R., Almasy, L., Koesterer, R., Smith, A. H., ... Farrer, L. A. (2014a). Genome-wide association study of alcohol dependence:significant findings in African- and European-Americans including novel risk loci. Molecular Psychiatry, 19(1), 41-49. Retrieved from http://www.pubmedcentral.nih.gov/ articlerender.fcgi?artid=4165335\&tool=pmcentrez\&rendertype $=$ abstract

Gelernter, J., Kranzler, H. R., Sherva, R., Koesterer, R., Almasy, L., Zhao, H., \& Farrer, L. A. (2014b). Genome-wide association study of opioid dependence: Multiple associations mapped to calcium and potassium pathways. Biological Psychiatry, 76(1), 66-74. Retrieved from http://www.pubmedcentral.nih.gov/articlerender.fcgi?artid= 3992201\&tool=pmcentrez\&rendertype=abstract

Gelernter, J., Sherva, R., Koesterer, R., Almasy, L., Zhao, H., Kranzler, H. R., \& Farrer, L. (2014c). Genome-wide association study of cocaine dependence and related traits: FAM53B identified as a risk gene. Molecular Psychiatry, 19(6), 717-723. Retrieved from http:// www.pubmedcentral.nih.gov/articlerender.fcgi?artid $=3865158 \&$ tool=pmcentrez\&rendertype $=$

Gesundheit, B., Rosenzweig, J. P., Naor, D., Lerer, B., Zachor, D. A., Procházka, V., ... Ashwood, P. (2013). Immunological and autoimmune considerations of Autism Spectrum Disorders. Journal of Autoimmunity, 44, 1-7. Retrieved from http://www.scopus.com/inward/record.url? eid=2-s2.0-84881375626\&partnerID=tZOtx3y1

Gibney, S. M., \& Drexhage, H. A. (2013). Evidence for a dysregulated immune system in the etiology of psychiatric disorders. Journal of Neuroimmune Pharmacology, 8(4), 900-920. Retrieved from http://www. ncbi.nlm.nih.gov/pubmed/23645137

Giynas Ayhan, M., Uguz, F., Askin, R., \& Gonen, M. S. (2014). The prevalence of depression and anxiety disorders in patients with euthyroid Hashimoto's thyroiditis: A comparative study. General Hospital Psychiatry, 36(1), 95-98. Retrieved from http://www.ncbi.nlm.nih.gov/ pubmed/24211158 
Goris, A., Boonen, S., D'hooghe, M.-B., \& Dubois, B. (2010). Replication of KIF21B as a susceptibility locus for multiple sclerosis. Journal of Medical Genetics, 47(11), 775-776.

Hartwig, F. P., Davies, N. M., Hemani, G., \& Davey Smith, G. (2016). Two-sample Mendelian randomization: Avoiding the downsides of a powerful, widely applicable but potentially fallible technique. International Journal of Epidemiology, 45(6), 1717-1726. Retrieved from http://www.ncbi.nlm.nih.gov/pubmed/28338968

Hegvik, T.-A., Instanes, J. T., Haavik, J., Klungsøyr, K., \& Engeland, A. (2017). Associations between attention-deficit/hyperactivity disorder and autoimmune diseases are modified by sex: A population-based cross-sectional study. European Child \& Adolescent Psychiatry, Retrieved from http://www.ncbi.nlm.nih.gov/pubmed/28983730

Hess, J. L., Tylee, D. S., Barve, R., de Jong, S., Ophoff, R. A., Kumarasinghe, N., ... Glatt, S. J. (2016). Transcriptome-wide mega-analyses reveal joint dysregulation of immunologic genes and transcription regulators in brain and blood in schizophrenia. Schizophrenia Research, 176(2-3), 114-124. Retrieved from http:// linkinghub.elsevier.com/retrieve/pii/S092099641630322X

Heyes, S., Pratt, W. S., Rees, E., Dahimene, S., Ferron, L., Owen, M. J., \& Dolphin, A. C. (2015). Genetic disruption of voltage-gated calcium channels in psychiatric and neurological disorders. Progress in Neurobiology, 134, 36-54. Retrieved from http://www.ncbi.nlm.nih.gov/ pubmed/26386135

Hibar, D. P., Stein, J. L., Renteria, M. E., Arias-Vasquez, A., Desrivières, S., Jahanshad, N., ... Medland, S. E. (2015). Common genetic variants influence human subcortical brain structures. Nature, 520(7546), 224-229. Retrieved from http://www.nature.com/articles/ nature14101

Hinds, D. A., McMahon, G., Kiefer, A. K., Do, C. B., Eriksson, N., Evans, D. M., ... Tung, J. Y. (2013). A genome-wide association meta-analysis of self-reported allergy identifies shared and allergy-specific susceptibility loci. Nature Genetics, 45(8), 907-911. Retrieved from http://www.pubmedcentral.nih.gov/articlerender.fcgi? artid $=3753407 \&$ tool $=$ pmcentrez\&rendertype $=$ abstract.

Hou, L., Bergen, S. E., Akula, N., Song, J., Hultman, C. M., Landén, M., ... McMahon, F. J. (2016). Genome-wide association study of 40,000 individuals identifies two novel loci associated with bipolar disorder. Human Molecular Genetics, 25(15), 3383-3394. Retrieved from http:// www.ncbi.nlm.nih.gov/pubmed/27329760

Instanes, J. T., Halmøy, A., Engeland, A., Haavik, J., Furu, K., \& Klungsøyr, K. (2017). Attention-deficit/hyperactivity disorder in offspring of mothers with inflammatory and immune system diseases. Biological Psychiatry, 81(5), 452-459. Retrieved from http://www. ncbi.nlm.nih.gov/pubmed/26809250

Jernigan, T. L., Brown, T. T., Hagler, D. J. Jr., Akshoomoff, N., Bartsch, H., Newman, E., ... Dale, A. M. (2016). The Pediatric Imaging, Neurocognition, and Genetics (PING) data repository. Neurolmage, 124(Pt B), 1149-1154. Retrieved from http://www.sciencedirect.com/science/ article/pii/S1053811915003572

Jones, K. A., \& Thomsen, C. (2013). The role of the innate immune system in psychiatric disorders. Molecular and Cellular Neurosciences, 53, 52-62. Retrieved from http://www.sciencedirect.com/science/ article/pii/S1044743112001844

Jostins, L., \& Ripke, S. (2012). Host-microbe interactions have shaped the genetic architecture of inflammatory bowel disease. Nature, 491(7422), 119-124. Retrieved from http://www.pubmedcentral.nih.gov/ articlerender.fcgi?artid $=3491803 \&$ tool=pmcentrez\&rendertype $=$ abstract

Jung, J.-Y., Kohane, I. S., \& Wall, D. P. (2011). Identification of autoimmune gene signatures in autism. Translational Psychiatry, 1, e63. Retrieved from http://www.scopus.com/inward/record.url?eid=2-s2. 0-83755167956\&partnerID=tZOtx3y1

Kiryluk, K., Li, Y., Scolari, F., Sanna-Cherchi, S., Choi, M., Verbitsky, M., ... Gharavi, A. G. (2014). Discovery of new risk loci for IgA nephropathy implicates genes involved in immunity against intestinal pathogens. Nature Genetics, 46(11), 1187-1196. Retrieved from http://www. pubmedcentral.nih.gov/articlerender.fcgi?artid=4213311\&tool= pmcentrez\&rendertype $=$ abstract.

Labonte, D., Thies, E., \& Kneussel, M. (2014). The kinesin KIF21B participates in the cell surface delivery of gamma2 subunit-containing
GABA-A receptors. European Journal of Cell Biology, 93(8-9), 338-346. Retrieved from https://doi.org/10.1016/j.ejcb.2014.07.007.

Lambert, J. C., Ibrahim-Verbaas, C. A., Harold, D., Naj, A. C., Sims, R., Bellenguez, C., ... Amouyel, P. (2013). Meta-analysis of 74,046 individuals identifies 11 new susceptibility loci for Alzheimer's disease. Nature Genetics, 45(12), 1452-1458. Retrieved from http://www. pubmedcentral.nih.gov/articlerender.fcgi?artid=3896259\&tool= pmcentrez\&rendertype $=$ abstract

Lee, S. H., Ripke, S., Neale, B. M., Faraone, S. V., Purcell, S. M., Perlis, R. H., ... Wray, N. R. (2013). Genetic relationship between five psychiatric disorders estimated from genome-wide SNPs. Nature Genetics, 45(9), 984-994. Retrieved from http://www.pubmedcentral.nih.gov/ articlerender.fcgi?artid $=3800159 \&$ tool $=$ pmcentrez\&rendertype $=$ abstract

Levine, A. J., Service, S., Miller, E. N., Reynolds, S. M., Singer, E. J., Shapshak, P., ... Freimer, N. (2012). Genome-wide association study of neurocognitive impairment and dementia in HIV-infected adults. American Journal of Medical Genetics Part B: Neuropsychiatric Genetics, 159B(6), 669-683. Retrieved from http://www.pubmedcentral.nih. gov/articlerender.fcgi?artid $=3418456 \&$ tool=pmcentrez\& rendertype $=$ abstract.

Li, M., Wu, D. D., Yao, Y. G., Huo, Y. X., Liu, J. W., Su, B., ... Luo, X. J. (2016). Recent positive selection drives the expansion of a schizophrenia risk nonsynonymous variant at SLC39A8 in Europeans. Schizophrenia Bulletin, 42(1), 178-190. Retrieved from http://www.ncbi.nlm.nih. gov/pubmed/26006263

Liu, J. Z., van Sommeren, S., Huang, H., Ng, S. C., Alberts, R., Takahashi, A., ... Weersma, R. K. (2015). Association analyses identify 38 susceptibility loci for inflammatory bowel disease and highlight shared genetic risk across populations. Nature Genetics, 47(9), 979-986. Retrieved from http://www.ncbi.nlm.nih.gov/pubmed/26192919

Lu, Q., Li, B., Ou, D., Erlendsdottir, M., Powles, R. L., Jiang, T., ... Zhao, H. (2017). A powerful approach to estimating annotation-stratified genetic covariance via GWAS summary statistics. American Journal of Human Genetics, 101(6), 939-964.

Lubke, G. H., Laurin, C., Amin, N., Hottenga, J. J., Willemsen, G., van Grootheest, G., ... Boomsma, D. I. (2014). Genome-wide analyses of borderline personality features. Molecular Psychiatry, 19(8), 923-929. Retrieved from http://www.pubmedcentral.nih.gov/articlerender.fcgi? artid $=3872258 \&$ tool=pmcentrez\&rendertype $=$ abstract

Lumb, J. H., Li, Q., Popov, L. M., Ding, S., Keith, M. T., Merrill, B. D., ... Carette, J. E. (2017). DDX6 represses aberrant activation of interferon-stimulated genes. Cell Reports, 20(4), 819-831. Retrieved from http://www.ncbi.nlm.nih.gov/pubmed/28746868

Luykx, J. J., Olde Loohuis, L. M., Neeleman, M., Strengman, E., Bakker, S. C., Lentjes, E., ... Ophoff, R. A. (2016). Peripheral blood gene expression profiles linked to monoamine metabolite levels in cerebrospinal fluid. Translational Psychiatry, 6(12), e983. Retrieved from http://www.ncbi.nlm.nih.gov/pubmed/27959337

Marrie, R. A., Walld, R., Bolton, J. M., Sareen, J., Walker, J. R., Patten, S. B., ... Bernstein, C. N. (2017a). Increased incidence of psychiatric disorders in immune-mediated inflammatory disease. Journal of Psychosomatic Research, 101, 17-23. Retrieved from https://www. sciencedirect.com/science/article/pii/S0022399917307353

Marrie, R. A., Walld, R., Bolton, J. M., Sareen, J., Walker, J. R., Patten, S. B., ... Bernstein, C. N. (2017b). Rising incidence of psychiatric disorders before diagnosis of immune-mediated inflammatory disease. Epidemiology and Psychiatric Sciences, 1-10. Retrieved from http://www.ncbi. nlm.nih.gov/pubmed/29098977

Maschietto, M., Tahira, A. C., Puga, R., Lima, L., Mariani, D., da Silveira Paulsen, B., ... Brentani, H. (2015). Co-expression network of neural-differentiation genes shows specific pattern in schizophrenia. BMC Medical Genomics, 8(1), 23. Retrieved from http:// bmcmedgenomics.biomedcentral.com/articles/10.1186/ s12920-015-0098-9

Masi, A., Quintana, D. S., Glozier, N., Lloyd, A. R., Hickie, I. B., \& Guastella, A. J. (2015). Cytokine aberrations in autism spectrum disorder: A systematic review and meta-analysis. Molecular Psychiatry, 20(4), 440-446. Retrieved from http://www.scopus.com/inward/record. url?eid=2-s2.0-84926168759\&partnerID=tZOtx3y1 
Mei, L., \& Nave, K.-A. (2014). Neuregulin-ERBB signaling in the nervous system and neuropsychiatric diseases. Neuron, 83(1), 27-49. Retrieved from http://www.ncbi.nlm.nih.gov/pubmed/24991953

Meyer, U. (2014). Prenatal poly(i:C) exposure and other developmental immune activation models in rodent systems. Biological Psychiatry, 75(4), 307-315. Retrieved from http://www.scopus.com/inward/record. url?eid=2-s2.0-84892609862\&partnerlD=tZOtx3y1

Mick, E., McGough, J., Deutsch, C. K., Frazier, J. A., Kennedy, D., \& Goldberg, R. J. (2014). Genome-wide association study of proneness to anger. PLoS One, 9(1), e87257. Retrieved from http://journals.plos. org/plosone/article?id=10.1371/journal.pone.0087257

Miller, A. H., Haroon, E., Raison, C. L., \& Felger, J. C. (2013). Cytokine targets in the brain: Impact on neurotransmitters and neurocircuits. Depression and Anxiety, 30(4), 297-306. Retrieved from http://www. scopus.com/inward/record.url?eid=2-s2.0-84876079221\& partnerID=tZOtx3y1

Modabbernia, A., Taslimi, S., Brietzke, E., \& Ashrafi, M. (2013). Cytokine alterations in bipolar disorder: A meta-analysis of 30 studies. Biological Psychiatry, 74(1), 15-25. Retrieved from http://www.ncbi.nlm.nih. gov/pubmed/23419545

Muller, Y. L., Hanson, R. L., Knowler, W. C., Fleming, J., Goswami, J., Huang, K., ... Baier, L. J. (2013). Identification of genetic variation that determines human trehalase activity and its association with type 2 diabetes. Human Genetics, 132, 697-707.

Murakami, A., Matsuda, M., Harada, Y., \& Hirata, M. (2017). Phospholipase C-related, but catalytically inactive protein (PRIP) up-regulates osteoclast differentiation via calcium-calcineurin-NFATc1 signaling. Journal of Biological Chemistry, 292(19), 7994-8006. Retrieved from http:// www.ncbi.nlm.nih.gov/pubmed/28341745

Nielsen, P. R., Benros, M. E., \& Dalsgaard, S. (2017). Associations between autoimmune diseases and attention-deficit/hyperactivity disorder: A Nationwide Study. Journal of the American Academy of Child \& Adolescent Psychiatry, 56(3), 234-240.e1. Retrieved from http://www.ncbi. nlm.nih.gov/pubmed/28219489

Ohn, J. H. (2017). The landscape of genetic susceptibility correlations among diseases and traits. Journal of the American Medical Informatics Association, 24(5), 921-926. Retrieved from http://www.ncbi.nlm.nih. gov/pubmed/28371808

Ohsawa, M., Okayama, A., Nakamura, M., Onoda, T., Kato, K., Itai, K., ... Hiramori, K. (2005). CRP levels are elevated in smokers but unrelated to the number of cigarettes and are decreased by long-term smoking cessation in male smokers. Preventive Medicine, 41(2), 651-656. Retrieved from http://www.ncbi.nlm.nih.gov/pubmed/15917065

Okada, Y., Wu, D., Trynka, G., Raj, T., Terao, C., Ikari, K., ... Plenge, R. M. (2014). Genetics of rheumatoid arthritis contributes to biology and drug discovery. Nature, 506(7488), 376-381. Retrieved from http:// www.pubmedcentral.nih.gov/articlerender.fcgi?artid $=3944098 \&$ tool=pmcentrez\&rendertype=abstract.

Oskvig, D. B., Elkahloun, A. G., Johnson, K. R., Phillips, T. M., \& Herkenham, M. (2012). Maternal immune activation by LPS selectively alters specific gene expression profiles of interneuron migration and oxidative stress in the fetus without triggering a fetal immune response. Brain, Behavior, and Immunity, 26(4), 623-634. Retrieved from http://www.scopus.com/inward/record.url?eid=2-s2. 0-84858740380\&partnerID=tZOtx3y1 [InsertedFromOnline]

Otowa, T., Hek, K., Lee, M., Byrne, E. M., Mirza, S. S., Nivard, M. G., ... Hettema, J. M. (2016). Meta-analysis of genome-wide association studies of anxiety disorders. Molecular Psychiatry, 21, 1391-9. Retrieved from http://www.nature.com/doifinder/10.1038/mp.2015.197.

Papp, G., Szabó, K., Szekanecz, Z., \& Zeher, M. (2014). Follicular helper T cells in autoimmune diseases. Rheumatology, 53(7), 1159-1160. Retrieved from https://academic.oup.com/rheumatology/article/1793107/ Follicular

Pego-Reigosa, J. M., \& Isenberg, D. A. (2008). Psychosis due to systemic lupus erythematosus: Characteristics and long-term outcome of this rare manifestation of the disease. Rheumatology (Oxford, England), 47(10), 1498-1502. Retrieved from http://www.scopus.com/inward/ record.url?eid=2-s2.0-52949096539\&partnerID=tZOtx3y1

Pickrell, J. K., Berisa, T., Liu, J. Z., Ségurel, L., Tung, J. Y., \& Hinds, D. A. (2016). Detection and interpretation of shared genetic influences on
42 human traits. Nature Genetics, 48(7), 709-717. Retrieved from http://www.nature.com/doifinder/10.1038/ng.3570

Porter, H. F., \& O'Reilly, P. F. (2017). Multivariate simulation framework reveals performance of multi-trait GWAS methods. Scientific Reports, 7, 38837. Retrieved from http://www.nature.com/articles/srep38837

Pouget, J. G., Han, B., Mignot, E., Ollila, H. M., Barker, J., Spain, S., ... Knight, J. (2016). Polygenic analysis of schizophrenia and 19 immune diseases reveals modest pleiotropy. Cold Spring Harbor Labs Journals. Retrieved from http://biorxiv.org/lookup/doi/10.1101/068684

Psychiatric GWAS Consortium Bipolar Disorder Working Group. (2011). Large-scale genome-wide association analysis of bipolar disorder identifies a new susceptibility locus near ODZ4. Nature Genetics, 43(10), 977-983. Retrieved from http://eutils.ncbi.nlm.nih.gov/entrez/eutils/ elink.fcgi?dbfrom=pubmed\&id $=21926972 \&$ retmode $=$ ref $\& \mathrm{cmd}=$ prlinks \%5Cnpapers3://publication/doi/10.1038/ng.943.

Radstake, T. R., Gorlova, O., Rueda, B., Martin, J. E., Alizadeh, B. Z., Palomino-Morales, R., ... Mayes, M. D. (2010). Genome-wide association study of systemic sclerosis identifies CD247 as a new susceptibility locus. Nature Genetics, 42(5), 426-429. Retrieved from http:// www.pubmedcentral.nih.gov/articlerender.fcgi?artid=2861917\& tool=pmcentrez\&rendertype $=$ abstract.

Rege, S., \& Hodgkinson, S. J. (2013). Immune dysregulation and autoimmunity in bipolar disorder: Synthesis of the evidence and its clinical application. Australian \& New Zealand Journal of Psychiatry, 47(12), 1136-1151. Retrieved from http://www.ncbi.nlm.nih.gov/ pubmed/23908311

Ripke, S. (2014). Biological insights from 108 schizophrenia-associated genetic loci. Nature, 511(7510), 421-427. Retrieved from https://doi. org/10.1038/nature13595

Ripke, S., Wray, N. R., Lewis, C. M., Hamilton, S. P., Weissman, M. M., Breen, G., ... Sullivan, P. F. (2013). A mega-analysis of genome-wide association studies for major depressive disorder. Molecular Psychiatry, 18(4), 497-511. Retrieved from http://www.pubmedcentral.nih.gov/ articlerender.fcgi?artid=3837431\&tool=pmcentrez\&rendertype=abstract

Schmunk, G., \& Gargus, J. J. (2013). Channelopathy pathogenesis in autism spectrum disorders. Frontiers in Genetics, 4, 222. Retrieved from http://www.ncbi.nlm.nih.gov/pubmed/24204377

Sekar, A., Bialas, A. R., de Rivera, H., Davis, A., Hammond, T. R., Kamitaki, N., ... Handsaker, R. E. (2016). Schizophrenia risk from complex variation of complement component 4. Nature, 530(7589), 177-183. Retrieved from http://www.ncbi.nlm.nih.gov/ pubmed/26814963\%5Cnhttp://www.pubmedcentral.nih.gov/ articlerender.fcgi?artid=PMC4752392.

Shatz, C. J. (2009). MHC class I: An unexpected role in neuronal plasticity. Neuron, 64(1), 40-45. Retrieved from http://www.scopus.com/ inward/record.url?eid=2-s2.0-70349792000\&partnerID=tZOtx3y1

Shi, H., Mancuso, N., Spendlove, S., \& Pasaniuc, B. (2017). Local genetic correlation gives insights into the shared genetic architecture of complex traits. The American Journal of Human Genetics, 101(5), 737-751. Retrieved from http://www.ncbi.nlm.nih.gov/pubmed/29100087

Sidhom, O., Laadhar, L., Zitouni, M., Ben Alaya, N., Rafrafi, R., Kallel-Sellami, M., \& Makni, S. (2012). Spectrum of autoantibodies in Tunisian psychiatric inpatients. Immunological Investigations, 41(5), 538-549. Retrieved from http://www.ncbi.nlm.nih.gov/pubmed/22612859

Silva, D., Colvin, L., Hagemann, E., Stanley, F., \& Bower, C. (2014). Children diagnosed with attention deficit disorder and their hospitalisations: Population data linkage study. European Child \& Adolescent Psychiatry, 23(11), 1043-1050. Retrieved from http://www.scopus.com/inward/ record.url?eid=2-s2.0-84901735027\&partnerID=tZOtx3y1

Sivertsen, B., Petrie, K. J., Wilhelmsen-Langeland, A., \& Hysing, M. (2014). Mental health in adolescents with Type 1 diabetes: Results from a large population-based study. BMC Endocrine Disorders, 14(1), 83. Retrieved from http://www.ncbi.nlm.nih.gov/pubmed/25303963

Sleiman, P. M. A., Wang, M. L., Cianferoni, A., Aceves, S., Gonsalves, N., Nadeau, K., ... Hakonarson, H. (2014). GWAS identifies four novel eosinophilic esophagitis loci. Nature Communications, 5, 5593. Retrieved from http://www.pubmedcentral.nih.gov/articlerender.fcgi? artid $=4238044 \&$ tool $=$ pmcentrez\&rendertype $=$ abstract.

Smith, S. E. P., Li, J., Garbett, K., Mirnics, K., \& Patterson, P. H. (2007). Maternal immune activation alters fetal brain development through interleukin-6. The Journal of Neuroscience : The Official Journal of the 
Society for Neuroscience, 27(40), 10695-10702. Retrieved from http://www.scopus.com/inward/record.url?eid=2-s2. 0-35148872226\&partnerID=tZOtx3y1

Solmi, M., Veronese, N., Favaro, A., Santonastaso, P., Manzato, E., Sergi, G., \& Correll, C. U. (2015). Inflammatory cytokines and anorexia nervosa: A meta-analysis of cross-sectional and longitudinal studies. Psychoneuroendocrinology, 51, 237-252. Retrieved from https://www. sciencedirect.com/science/article/pii/S0306453014003795

Stringer, S., Kahn, R. S., de Witte, L. D., Ophoff, R. A., \& Derks, E. M. (2014). Genetic liability for schizophrenia predicts risk of immune disorders. Schizophrenia Research, 159(2-3), 347-352. Retrieved from http://www. scopus.com/inward/record.url?eid=2-s2.0-84922450900\& partnerID=tZOtx3y1

Takenaka, K., Fukami, K., Otsuki, M., Nakamura, Y., Kataoka, Y., Wada, M., ... Takenawa, T. (2003). Role of phospholipase C-L2, a novel phospholipase C-like protein that lacks lipase activity, in B-cell receptor signaling. Molecular and Cellular Biology, 23(20), 7329-7338. Retrieved from http://www.ncbi.nlm.nih.gov/pubmed/14517301

Tapper, W., Jones, A. V., Kralovics, R., Harutyunyan, A. S., Zoi, K., Leung, W., ... Cross, N. C. P. (2015). Genetic variation at MECOM, TERT, JAK2 and HBS1L-MYB predisposes to myeloproliferative neoplasms. Nature Communications, 6(1), 6691., Retrieved from http:// www.nature.com/doifinder/10.1038/ncomms7691.

The Network and Pathway Analysis Subgroup of the Psychiatric Genomics Consortium. (2015). Psychiatric genome-wide association study analyses implicate neuronal, immune and histone pathways. Nature Neuroscience, 18(2), 199-209. Retrieved from https://doi.org/10.1038/nn.3922

The Tobacco and Genetics Consortium. (2010). Genome-wide meta-analyses identify multiple loci associated with smoking behavior. Nature Genetics, 42(5), 441-447. Retrieved from http://www.nature. com/ng/journal/v42/n5/full/ng.571.html\#corres-auth.

Tiosano, S., Farhi, A., Watad, A., Grysman, N., Stryjer, R., Amital, H., \& Amital, D. (2016). Schizophrenia among patients with systemic lupus erythematosus: Population-based cross-sectional study. Epidemiology and Psychiatric Sciences, 26, 424-429.

Toyoda, H., Saito, M., Sato, H., Tanaka, T., Ogawa, T., Yatani, H., ... Kang, Y. (2015). Enhanced desensitization followed by unusual resensitization in GABAA receptors in phospholipase C-related catalytically inactive protein-1/2 double-knockout mice. Pflügers Archiv - European Journal of Physiology, 467(2), 267-284. Retrieved from http://www. ncbi.nlm.nih.gov/pubmed/24737248

Tsoi, L. C., Spain, S. L., Ellinghaus, E., Stuart, P. E., Capon, F., Knight, J., ... Elder, J. T. (2015). Enhanced meta-analysis and replication studies identify five new psoriasis susceptibility loci. Nature Communications, 6, 7001. Retrieved from http://www.nature.com/ ncomms/2015/150427/ncomms8001/full/ncomms8001.html

Tung, J. Y., Do, C. B., Hinds, D. A., Kiefer, A. K., Macpherson, J. M., Chowdry, A. B., ... Eriksson, N. (2011). Efficient Replication of over 180 Genetic Associations with Self-Reported Medical Data P. H. Reitsma, ed. PLoS One, 6(8), e23473. Retrieved from http://dx.plos. org/10.1371/journal.pone.0023473

Turley, P., Walters, R. K., Maghzian, O., Okbay, A., Lee, J. J., Fontana, M. A., ... Benjamin, D. J. (2018). Multi-trait analysis of genome-wide association summary statistics using MTAG. Nature Genetics, 50, 229-237. Retrieved from http://www.nature.com/ articles/s41588-017-0009-4
Vaeth, M., Müller, G., Stauss, D., Dietz, L., Klein-Hessling, S., Serfling, E., ... Berberich-Siebelt, F. (2014). Follicular regulatory T cells control humoral autoimmunity via NFAT2-regulated CXCR5 expression. The Journal of Experimental Medicine, 211(3), 545-561. Retrieved from http://www.ncbi.nlm.nih.gov/pubmed/24590764

Vogel, S. W. N., Bijlenga, D., Verduijn, J., Bron, T. I., Beekman, A. T. F. Kooij, J. J. S., \& Penninx, B. W. J. H. (2017). Attention-deficit/hyperactivity disorder symptoms and stress-related biomarkers. Psychoneuroendocrinology, 79, 31-39. Retrieved from http://www.ncbi.nlm.nih.gov/pubmed/ 28249186

Wang, Q., Yang, C., Gelernter, J., \& Zhao, H. (2015). Pervasive pleiotropy between psychiatric disorders and immune disorders revealed by integrative analysis of multiple GWAS. Human Genetics, 134(11-12), 1195-1209. Retrieved from http://link.springer.com/10.1007/ s00439-015-1596-8

Wray, N. R., Lee, S. H., \& Kendler, K. S. (2012). Impact of diagnostic misclassification on estimation of genetic correlations using genome-wide genotypes. European Journal of Human Genetics, 20(6), 668-674. Retrieved from https://doi.org/10.1038/ejhg.2011.257

Xie, G., Roshandel, D., Sherva, R., Monach, P. A., Lu, E. Y., Kung, T., .. Siminovitch, K. A. (2013). Association of granulomatosis with polyangiitis (Wegener's) with HLA-DPB1*04 and SEMA6A gene variants: Evidence from genome-wide analysis. Arthritis and Rheumatism, 65(9), 2457-2468.

Xu, B., Roos, J. L., Dexheimer, P., Boone, B., Plummer, B., Levy, S., .. Karayiorgou, M. (2011). Exome sequencing supports a de novo mutational paradigm for schizophrenia. Nature Genetics, 43(9), 864-868. Retrieved from http://www.ncbi.nlm.nih.gov/pubmed/21822266

Yuce, M., Guner, S. N., Karabekiroglu, K., Baykal, S., Kilic, M., Sancak, R., \& Karabekiroglu, A. (2014). Association of Tourette syndrome and obsessive-compulsive disorder with allergic diseases in children and adolescents: A preliminary study. European Review for Medical and Pharmacological Sciences, 18(3), 303-310.

Zheng, J., Erzurumluoglu, A. M., Elsworth, B. L., Kemp, J. P., Howe, L., Haycock, P. C., ... Neale, B. M. (2016). LD Hub: A centralized database and web interface to perform LD score regression that maximizes the potential of summary level GWAS data for SNP heritability and genetic correlation analysis. Bioinformatics, 33(2), 272-279. Retrieved from http://biorxiv.org/content/early/2016/05/03/051094.abstract

\section{SUPPORTING INFORMATION}

Additional supporting information may be found online in the Supporting Information section at the end of the article.

How to cite this article: Tylee DS, Sun J, Hess JL, et al. Genetic correlations among psychiatric and immune-related phenotypes based on genome-wide association data. Am J Med Genet Part B. 2018;177B:641-657. https://doi.org/10. 1002/ajmg.b.32652 